\title{
A Hybrid Genetic Algorithm and Sperm Swarm Optimization (HGASSO) for Multimodal Functions
}

\author{
Hisham A. Shehadeh, Faculty of Computer Science and Informatics, Amman Arab University, Jordan* \\ (iD) https://orcid.org/0000-0003-4054-6561 \\ Hossam M. J. Mustafa, Faculty of Computer Science and Informatics, Amman Arab University, Jordan \\ (iD) https://orcid.org/0000-0003-2015-7497
}

Mohammad Tubishat, School of Computing and Technology, Asia Pacific University of Technology and Innovation, Malaysia

\begin{abstract}
In this paper, the authors propose a hybrid algorithm combining two different metaheuristic methods, genetic algorithms (GA) and sperm swarm optimization (SSO), for the global optimization of multimodal benchmarks functions. The proposed hybrid genetic algorithm and sperm swarm optimization (HGASSO) operates based on incorporates concepts from GA and SSO which generates individuals in a new iteration not only by crossover and mutation operations as proposed in GA but also by techniques of local search of SSO. The main idea behind this hybridization is to reduce the probability of trapping in local optimum of multi-modal problem. The algorithm is compared against GA and SSO metaheuristic optimization algorithms. The experimental results using a suite of multimodal benchmarks functions taken from the literature have evinced the superiority of the proposed HGASSO approach over the other approaches in terms of quality of results and convergence rates which obtained good results in solving the multimodal benchmarks functions including cosine, sine, and exponent in their formulation.
\end{abstract}

\section{KEYWORDS}

Evolutionary Method, Genetic Algorithms (GA), Hybrid Algorithm, Multimodal Benchmarks Functions, Sperm Swarm Optimization (SSO), Swarm Intelligence Algorithms

\section{INTRODUCTION}

During the last decades, there has been a huge growing in the area of evolutionary computing, which has indicated modern techniques for solving various types of optimization problems. In contrast to classical or traditional optimization approaches, which emphasize exact and accurate computation, but these approaches may fall down in obtaining the solution of a global optimum. Based on that, evolutionary computation emerged to provide more efficient and robust technique for solving complex problems (Shehadeh et al. 2018a; Fogel, 2005). "Genetic algorithm (GA)" is one of the most prevalent branches between the existing evolutionary approaches. GA is considered as an inherently discrete stochastic search approach depends on the techniques of generating genetics, natural selection, and 
evolution (Shehadeh et al, 2018a; Paulinas and Ušinskas, 2007). Since this type of metaheuristics simultaneously can search and evaluate many sites in the domain of problem, which is more likely to discover the global optimum of an optimization problem. Furthermore, it easy to implement and use, which uses a measure of performance of simple scalar that does not use or require derivative information (Shehadeh et al., 2018a).

More recently, Hisham A. Shehadeh et al. (Shehadeh et al., 2017; Shehadeh et al. 2018b, Shehadeh et al. 2018c) proposed a new metaheuristic method, namely "Sperm Swarm Optimization" (denoted as SSO). The idea of this approach is inspired by the behaviors of sperm swarm through the procedure of natural fertilization. The theory of SSO represents a solution process, which each sperm swims through the domain of multidimensional search space while the sperm's position and velocity are constantly updated based on the previous position of the sperm, as well as the best performance of the swarm in the entire population.

In contrast with GA, SSO is an inherently continuous approach in which has various attractive features. It has memory, so the prior knowledge can be retained by all the sperms in each generation; whereas in GA, the scenario is different, which is considered as an inherently discrete approach, so the prior knowledge of the problem is discarded each iteration by reserving the best individuals and eliminating the worst individuals at each generation. To date, SSO has been successfully applied to generate the optimal solution for different continuous nonlinear functions in practice (Shehadeh et al., 2018c), but until recently it had not been hybridized to deal with multimodal problems. SSO seems particularly appropriate for multimodal tasks mainly because of the good quality of solutions and the high speed of convergence that the algorithm shows for solving different kinds of single-objective problems (Shehadeh et al., 2018c).

There are many studies that have been investigated the hybridization of evolutionary algorithms with local search. We can summarize them as follows:

Soleimani et al. proposed a hybrid approach that integrates "Particle Swarm Optimization (PSO)" with GA. The proposed approach was used to optimize problems of supply chain network. The results showed that the proposed approach has better convergence and quality of solution than GA (Soleimani \& Kannan, 2015).

On the other hand, Samuel et al. suggested new optimization approach that merges the functionality of PSO and GA. The proposed approach was used to solve the scheduling problem of power generator. The results proved the efficiency of the proposed approach in finding solution for the aforementioned problem (Samuel \& Rajan, 2015).

In different view, Fang et al. proposed a new hybrid approach that integrates "Artificial Fish Swarm Algorithm (AFSA)" with GA. This approach was used to solve scheduling problem of the hydrothermal systems. To prove the performance of the proposed approach, Fang et al. tested this approach on two hydrothermal systems (Fang et al., 2014).

In a different work, Kao et al. proposed a hybrid approach that integrates PSO with GA. The proposed approach was applied to solve 17 multi-modal functions. The results showed that the proposed approach has better convergence and quality of solution than other approaches (Kao \& Zahara, 2008).

Gholami et al. discussed a hybrid approach that merges the functionality of PSO with GA. They used this approach to optimize bank shape problem. The result showed the performance of the approach under different scenarios (Gholami et al., 2018).

On the other hand, Gacem et al. proposed a hybrid approach that integrates the functionality of GA and PSO. The proposed approach is applied to optimize the power flow problem. The results showed that the proposed approach has better convergence and quality of solution than GA and PSO (Gacem \& Benattous, 2017).

In a later study, Chaudhary et al. proposed a hybrid approach that combines the functionality of GA and "Gravitational Search Algorithm (GSA)". They used this approach to optimize the load scheduling problem in cloud computing (Chaudhary \& Kumar, 2019). 
As mentioned in the aforementioned studies, the hybridization is always referred to the concept of memetic algorithm in which the procedures of two or more algorithms are merged as one procedure to solve a problem. Taking feature of the compensatory property of SSO and GA, in this paper, we propose a new method that merges evolutionary natures of SSO and GA. The proposed "Hybrid Genetic Algorithm and Sperm Swarm Optimization (HGASSO)" will be evaluated against a set of multimodal bed problems gathered from (Kao \& Zahara, 2008; Surjanovic \& Bingham, 2013) and the results are compared extensively with the original GA and SSO.

This paper is organized as follows: background on "Genetic Algorithm (GA)" and "Sperm Swarm Optimization (SSO)" is summarized in Sect. 2. Section 3 shows "Hybrid Genetic Algorithm and Sperm Swarm Optimization (HGASSO)". Section 4 summarizes experimental results. Sect. 5 summarizes the discussion. This work is concluded in Sect. 6.

\section{BACKGROUND ON GENETIC ALGORITHMS (GA) AND SPERM SWARM OPTIMIZATION (SSO)}

This section constructs the need for the study and gives a general background related to "Genetic Algorithm (GA)" and "Sperm Swarm Optimization (SSO)". In addition, this section presents view in-depth at pseudocodes of these optimization algorithms and their concepts.

\section{GENETIC ALGORITHMS (GA)}

GA is proposed by Holland in 1993, which refers to Biology Inspired Search (BIS) method that is inspired by Darwinian's theory of evolution (Goldberg \& Holland, 2002). GA has been successfully applied to solve different complex and nonlinear problems and proved its eligibilities over many classical optimization approaches, specifically when the problem under study has solutions of many local optimums. Each solution in GA is commonly formalized as a binary string, namely a chromosome. Usually, there is a fitness function has been used to evaluate this chromosome after the chromosome has been formalized. Upon accomplishment of the estimation, an operation called roulette wheel selection is utilized to choose pairs of better chromosomes randomly from the population to utilize such genetic operations as mutation as well as crossover that mimic natural procedure. The weak chromosome will be discarded from the population each time run. This evaluation procedure continues until the end criterion is achieved. The GA pseudocode is summarized in Algorithm 1 (Langdon \& Poli, 2002).

Algorithm 1 Genetic Algorithm (GA)

Begin

Step 1: Set the problem based on a genetic representation.

Step 2: Initialize population $p(0)=x_{1}^{0}, \ldots x_{N}^{0}$.

Step 3: Calculate the average fitness $F=\sum_{i}^{N} F\left(z_{i}\right) / N$ and give a

normalized fitness value for each individual $F\left(z_{i}^{t}\right) / N$.

Step 4: Give every $z_{i}$ a probability $p\left(z_{j}, t\right)$ based on its fitness.

Based on that, select $N$ vectors from $P(t)$. This provides the set $S(t)$.

Step 5: Pair values in $S(t)$ at random forming $N / 2$ pairs. Perform crossover with probability pcross to each pair. Perform mutation to form a new population $P(t+1)$.

step 6: Set $t=t+1$, go to step 3 .

End. 


\section{SPERM SWARM OPTIMIZATION (SSO)}

"Sperm Swarm Optimization (SSO)" is one of the latest optimization approaches developed by Hisham Shehadeh et al. (Shehadeh et al., 2017; Shehadeh et al. 2018b, Shehadeh et al. 2018c). SSO concept is depends on a metaphor of the procedure of natural fertilization of sperm swarm. Sperms represent as candidate solutions in the SSO approach, which swim around in the multidimensional search space domain. The position of each sperm is adjusted based on the previous position and the global best solution of the swarm. The global best solution represents the closest sperm to the egg, which is called the winner. The winner is depicted in Figure 1. Sperm movement affects by many limitations of reproductive system of female. These limitations can be summarized in the following points (Shehadeh et al., 2018a; Shehadeh et al., 2017; Shehadeh et al. 2018b, Shehadeh et al. 2018c):

- $\mathrm{pH}$ value, which is a random number between 7-14.

- Temperature value, which is a random number between 35.1-38.5.

The sperm adjust its velocity based on three rules. These rules can be summarized as follows:

- Initial velocity of the sperm, which is affected by $\mathrm{pH}$ value of the area.

- Personal best solution of the sperm, which is affected by $\mathrm{pH}$ and temperature values of the reached location. This velocity will be adjusted in the memory just if the current location is better than the old one.

- Global best solution, which is affected by $\mathrm{pH}$ and temperature values of the visited location. This velocity is recorded by the closest sperm to the egg, which is called the winner.

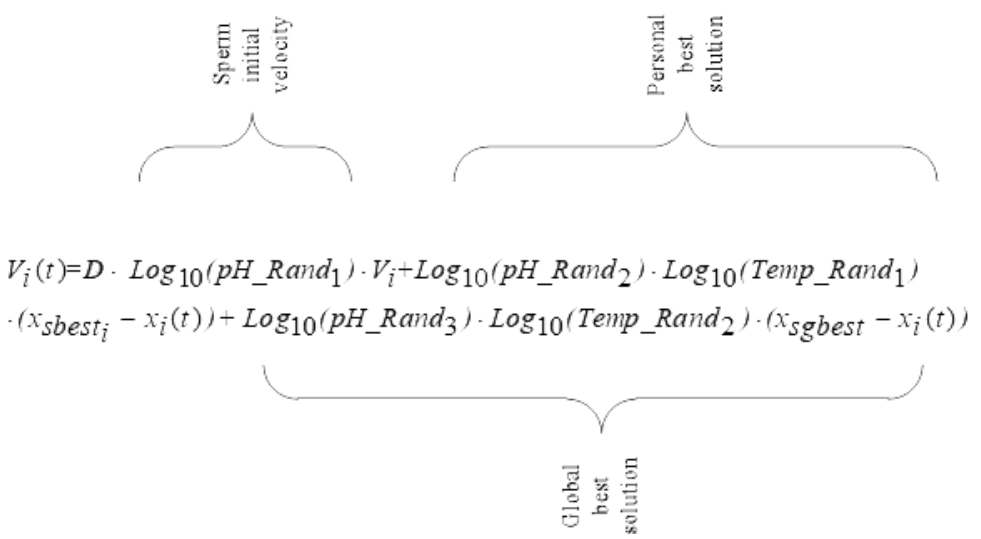

where,

- $\quad D$-factor of velocity damping, which is a random value between 0 and 1 ;

- $\quad p H \_R a n d, p H \_R a n d$, and $p H \_$Rand $_{3}$ - the $\mathrm{pH}$ value of visited area, which is a random value between 7-14;

- Temp_Rand, Temp_Rand 2 - the temperature value of visited area, which is a random value between 35.1-38.5;

- $\quad v_{i}$-velocity of sperm;

- $x_{i}$ - current position of sperm;

- $\quad x_{\text {sbest }}$ - personal best location of sperm $i$;

- $x_{\text {sgbest }}-$ global best location of the swarm.

The current best solution can be calculated by the following formula: 
Figure 1. The sperm swarm and winner

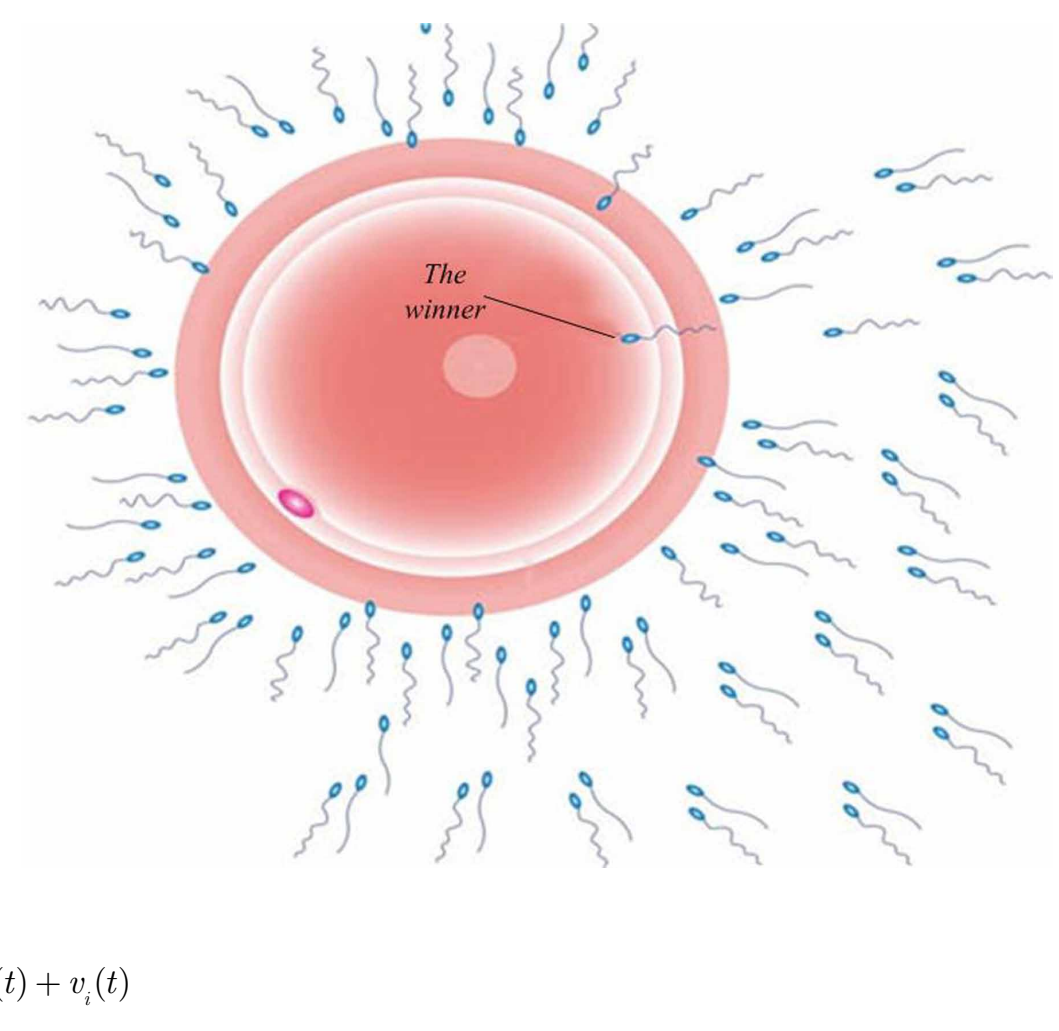

The full procedure of "Sperm Swarm Optimization (SSO)" approach can be summarized as follows (Shehadeh et al., 2018b; Shehadeh et al., 2018c):

Algorithm 2 sperm Swarm Optimization (SSO)

Begin

Step 1: For the each sperm, initialize location.

Step 2: for $i=1$ : size of population do

Step 3: evaluate the fitness for swarm

if obtained fitness $>x_{\text {sbest }}$ then

Assign the current value as the $x_{\text {sbest }}$

end if

end for

Step 4: appoint the $x_{\text {sqbest }}$ based on the winner.

Step 5: for $i=1$ : size of population do Apply Eq (1)

Apply Eq(2)

end for

Step 6: while maximum criterion is not reached go to step 2 End.

\section{HYBRID GENETIC ALGORITHM AND SPERM SWARM OPTIMIZATION (HGASSO)}

Usually, the main idea of the hybrid optimization algorithm can be considered as applying a local search approach with the genetic algorithm operations such as crossover and mutation in one procedure 
(Pomar et al., 2017; Moin et al. 2015). This section summarizes the structure of our proposed algorithm (denoted by HGASSO). From the pseudocode of algorithm 3, we can notice that GA and SSO both evaluate the same initial population. The individuals of the population may be represented as sperms in the case of SSO, or as chromosomes in the case of GA. In the proposed HGASSO, the local search is applied first with all the individuals, which includes the process of selecting the global best sperm (the winner), the process of selecting the personal best solution of the sperm, and the process of applying the sperm velocity update rule (Equation 1). This is followed by evaluating a fitness function. The crossover operator of the real-coded GA comes at this stage to have the site on the procedure, which is applied to all the individuals based on a crossover probability to prepare them for the mutation operation stage. The operator of GA, called mutation is used after the crossover operation to adjust an candidate solution with a value based on a random selection in the problem's space domain. The mutation is operated depends on a mutation probability that is determined at the beginning of the procedure. The full procedure of "Hybrid Genetic Algorithm and Sperm Swarm Optimization (HGASSO)" can be summarized as follows:

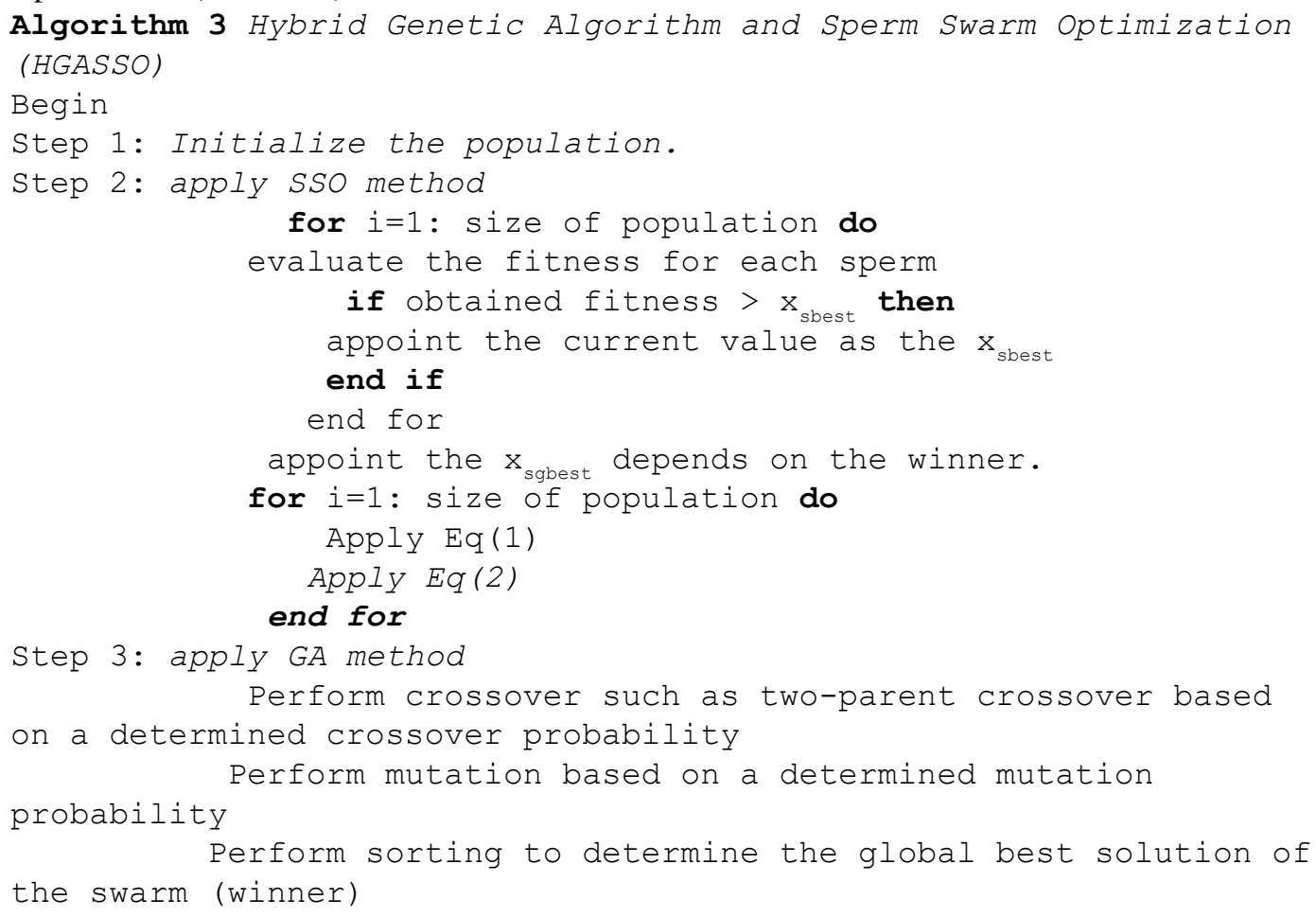

In this procedure, the crossover and mutation are applied to help the local search method to skip the local minima easily. Crossover and mutation help to make diversity in the population in the range of the problem's space domain. This is very important to be applied, especially with multimodal problems that contain multi-local extremes. Based on pseudocode of Algorithm 3, the algorithm sorts the population to determine the global best solution of the swarm. Therefore, the complexity of HGASSO is equal to $\mathrm{O}(\mathrm{n} \log \mathrm{n})$.

\section{EXPERIMENT AND RESULTS}

In order to compare the proposed HGASSO with both GA and SSO, we apply two assessments, including, quantitative and qualitative tests. For the quantitative test, we measure the best fitness, 
standard deviation, median, maximum value and average value that are achieved by the approaches for each benchmark function. Furthermore, we adopt a statistical test, namely "one-way ANOVA (Tukey's test)" to compare between approaches. This will be discussed later in Tables from 2 to 14, while for the qualitative test, we compare between the solution set that is achieved by our approach and the solution set that achieved by the other approaches. This refers to the concept of algorithm convergence, which is utilized to measure the ability of an algorithm to reach the optimal solution of a problem within a determined number of iterations. This will be discussed later in Figures from 12 to 22 .

These assessments are measured based on several well-known multimodal benchmark functions. Benchmark model is a nonlinear problem, which is utilized to estimate and validate the metaheuristic approaches (Krzeszowski \& Wiktorowicz, 2016). These functions are selected carefully as they are considered as standard bed test problem for estimating the optimization methods (Kaipa \& Ghose, 2017; Adorio \& Diliman, 2005; Molga \& Smutnicki, 2005; Back, 1996). The listed below benchmarks functions have several local extremes and all of them are minimization models, which their results should be minimum. The following points summarize the benchmarks functions that are used in this work:

\section{- Rastrigin Function}

The search space domain of this function is usually estimated on the hypercube $x_{i} \in[-5.12,5.12]$, $\forall i=1, \ldots, d$. Rastrigin problem has multiple local minima regularly distributed in its search space domain as depicted in Figure 2. The mathematical formulation of this problem is shown in Equation 3 (Surjanovic \& Bingham, 2013).

$$
f(y)=10 d+\sum_{i=1}^{d}\left[y_{i}^{2}-10 \cos \left(2 \pi y_{i}\right)\right]
$$

\section{- Ackley Function:}

Ackley function is considered as a very complex problem in which many metaheuristics methods are trapped in one of its multiple local minima. The optimal value of this function is 0 with a single global minimum at $(0, \ldots, 0)$, as depicted in Figure 3 . The mathematical formulation of this problem is shown in Equation 4. This problem can be estimated on the hypercube $x_{i} \in[-32.768,32.768] \forall i$ $=1, \ldots, d$. The recommended values of the parameters $\mathrm{c}, \mathrm{b}$ and a are $2 \pi, 0.2$, and 20 respectively (Surjanovic \& Bingham, 2013).

$$
f(y)=-a \exp \left(-\mathrm{b} \sqrt{\frac{1}{d} \sum_{i=1}^{d} y_{i}^{2}}\right)-\exp \left(\frac{1}{d} \sum_{i=1}^{d} \cos \left(c y_{i}\right)\right)+a+\exp (1)
$$

\section{- Bukin Function:}

This problem is always estimated on the rectangle $x_{1} \in[-15,-5], x_{2} \in[-3,3]$. Bukin function has a single global minimum at $(-10,1)$ and many local minima with an optimal value of 0 . Figure 4 
Figure 2. Rastrigin function

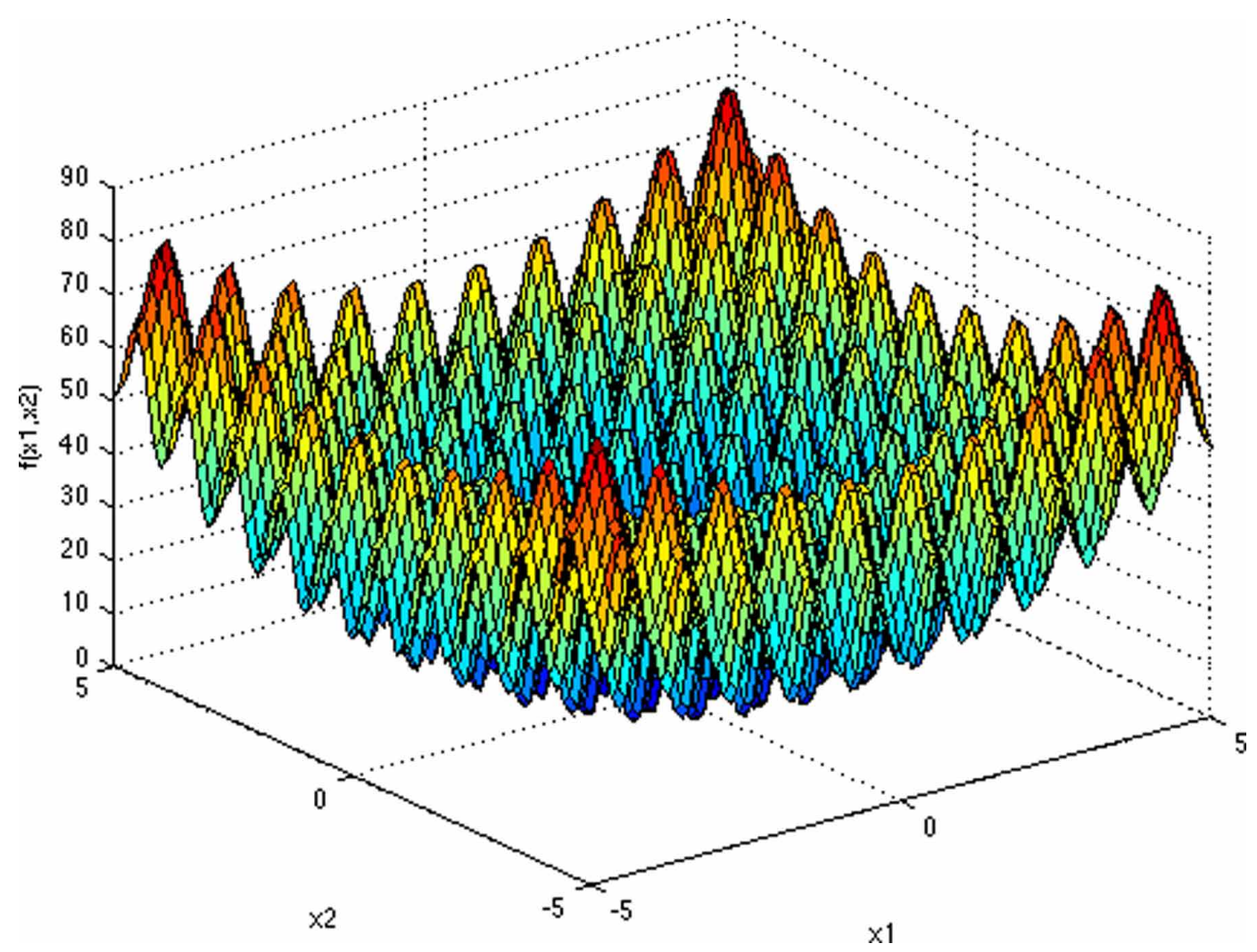

shows its local extremes, which lie in a series of ridges. The mathematical formulation of this problem is shown in Equation 5 (Surjanovic \& Bingham, 2013).

$$
f(y)=100 \sqrt{\left|y_{2}-0.01 y_{1}^{2}\right|}+0.01\left|y_{1}+10\right|
$$

\section{- Griewank Function}

Griewank model has many widespread local minima in its search space domain. This problem has a global minimum at $(0,0)$ with an optimal value of 0 . Griewank function is depicted in Figure 5 , which the zoomed-in plots present its complexity. This problem is always estimated on the hypercube $x_{i} \in[-600,600], \forall i=1, \ldots, \mathrm{d}$. The mathematical formulation of this problem can be described as in Equation 6 (Surjanovic \& Bingham, 2013).

$$
f(y)=\sum_{i=1}^{d} \frac{y_{i}^{2}}{4000}-\prod_{i=1}^{d} \cos \left(\frac{y_{i}}{\sqrt{i}}\right)+1,
$$




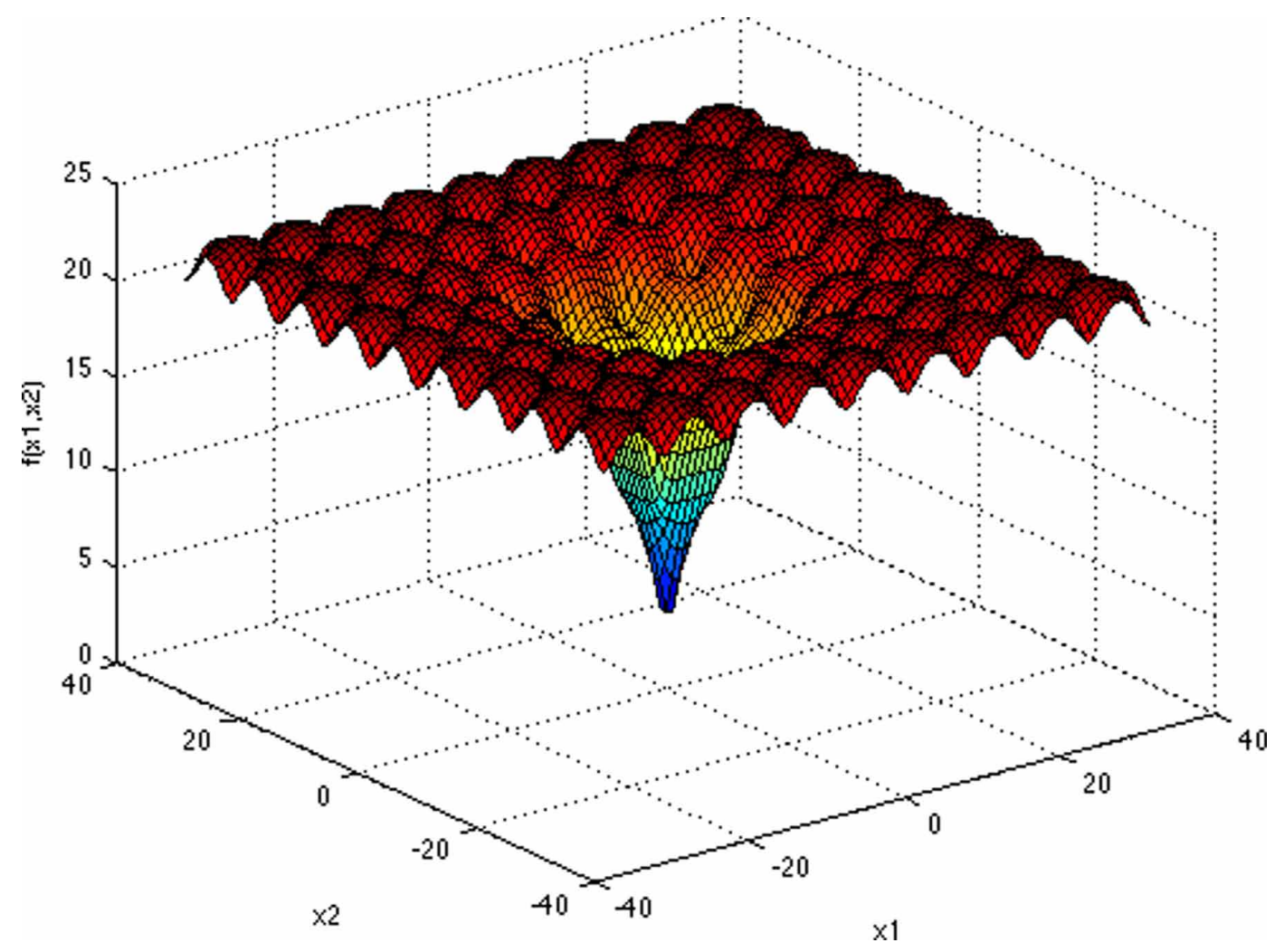

\section{- Cross-In-Tray Function}

Cross-In-Tray function has an optimal value of -2.06261 with several global minima at (1.3491, $-1.3491),(-1.3491,1.3491),(-1.3491,-1.3491)$, and $(1.3491,1.3491)$. This function is depicted in Figure 6 in which the right plot shows its characteristic of a cross with its several global minima. This problem is mainly estimated on the square $x_{i} \in[-10,10], \forall i=1,2$. The mathematical formulation of Cross-In-Tray can be represented as in Equation 7 (Surjanovic \& Bingham, 2013).

$$
f(y)=-0.0001\left(\mid \sin \left(y_{1}\right) \sin \left(y_{2}\right) \exp \left(\left|100-\frac{\sqrt{y_{1}^{2}+y_{2}^{2}}}{\pi}\right|\right)+1\right)^{0.1}
$$

\section{- Levy Function N.13}

The input values of this benchmark problem are commonly estimated on the square $x_{i} \in[-10$, 10], $\forall i=1,2$. This problem has a single global minimum at $(1,1)$ with an optimal value of 0 as depicted in Figure 7. The mathematical formulation of Levy function N.13 can be described as in Equation 8 (Surjanovic \& Bingham, 2013). 
Figure 4. Bukin function

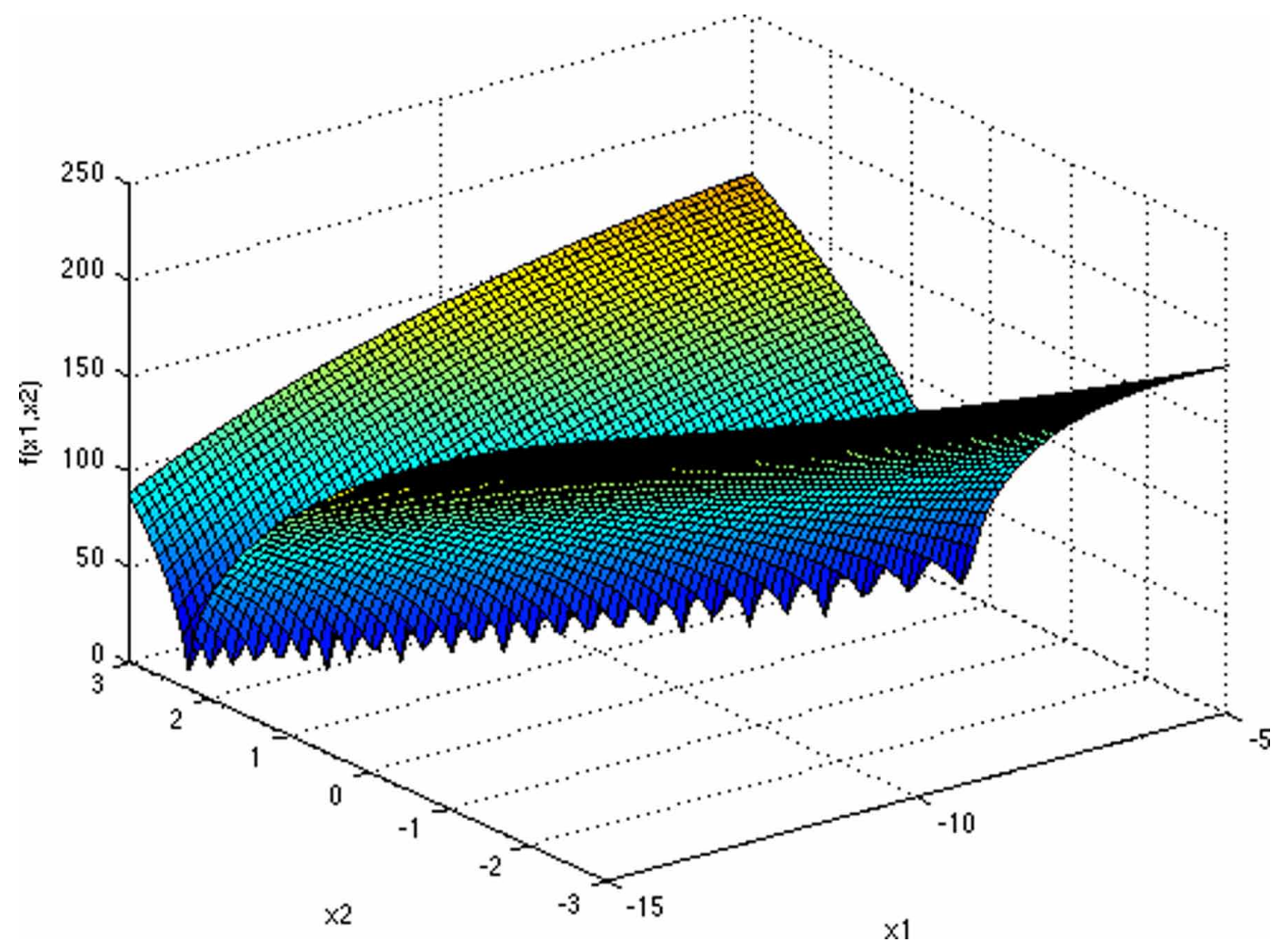

$f(y)=\sin ^{2}\left(3 \pi y_{1}\right)+\left(y_{1}-1\right)^{2}\left[1+\sin ^{2}\left(3 \pi y_{2}\right)\right]+\left(y_{2}-1\right)^{2}\left[1+\sin ^{2}\left(2 \pi y_{2}\right)\right]$,

\section{- Branin}

This benchmark is usually estimated on the square $x_{1} \in[-5,10], x_{2} \in[0,15]$, as shown in Figure 8. The optimal value of this function is 0.39789 with three local minima values at $(-3.14,12.275)$, $(3.14,2.275)$, and $(9.42478,2.475)$. The mathematical representation of Branin can be represented as in Equation 9 (Surjanovic \& Bingham, 2013).

$f(y)=\frac{1}{51.95}\left[\left(-\overline{y_{2}}-\frac{5 \cdot \bar{y}_{1}}{4 \pi^{2}}+\frac{5 \bar{y}_{1}}{\pi}-6\right)^{2}+\left(10-\frac{10}{8 \pi}\right) \cos \left(\bar{y}_{1}\right)-44.81\right]$

Where,

$\overline{y_{1}}=15 \bar{y}_{1}-5$,
$\bar{y}_{2=15 \bar{y}_{2}}$ 

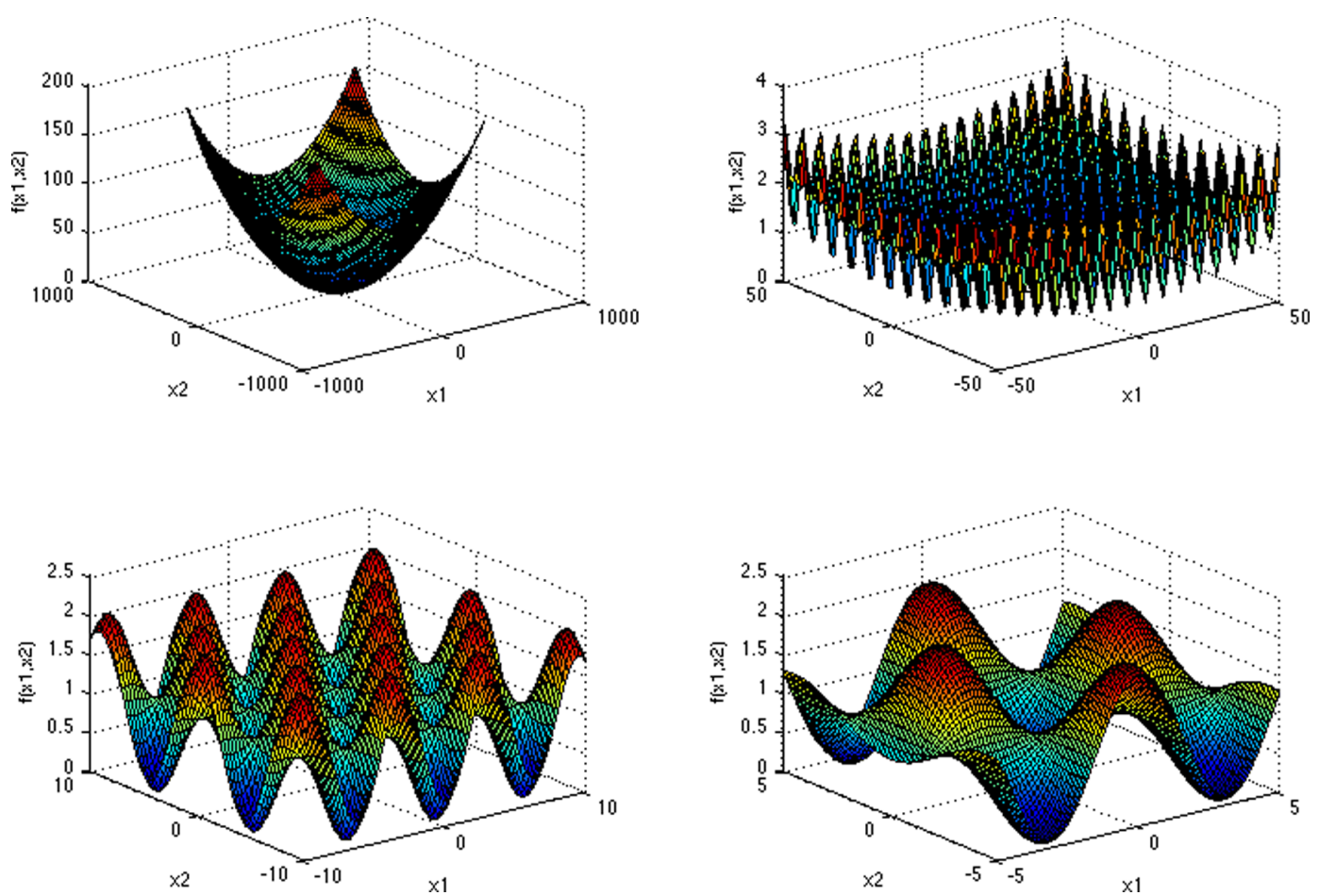

Figure 6. Cross-In-Tray function
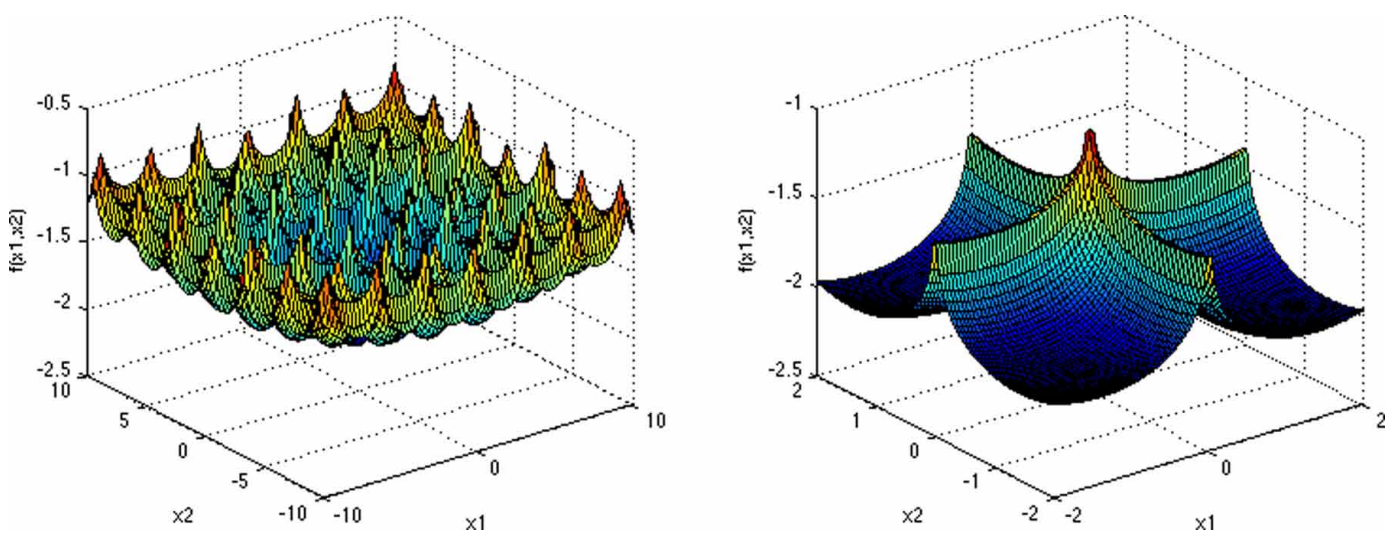

\section{- $\quad \operatorname{Easom}(\mathbf{E S})$}

This benchmark is mainly estimated on the square $x_{i} \in[-100,100], \forall i=1,2$, as shown in Figure 9. The Easom problem has several local minima with a single global minima at $(3.14,3.14)$. The optimal value of this function is -1. The mathematical formulation of Easom can be described as in Equation 10 (Surjanovic \& Bingham, 2013). 
Figure 7. Levy function N.13

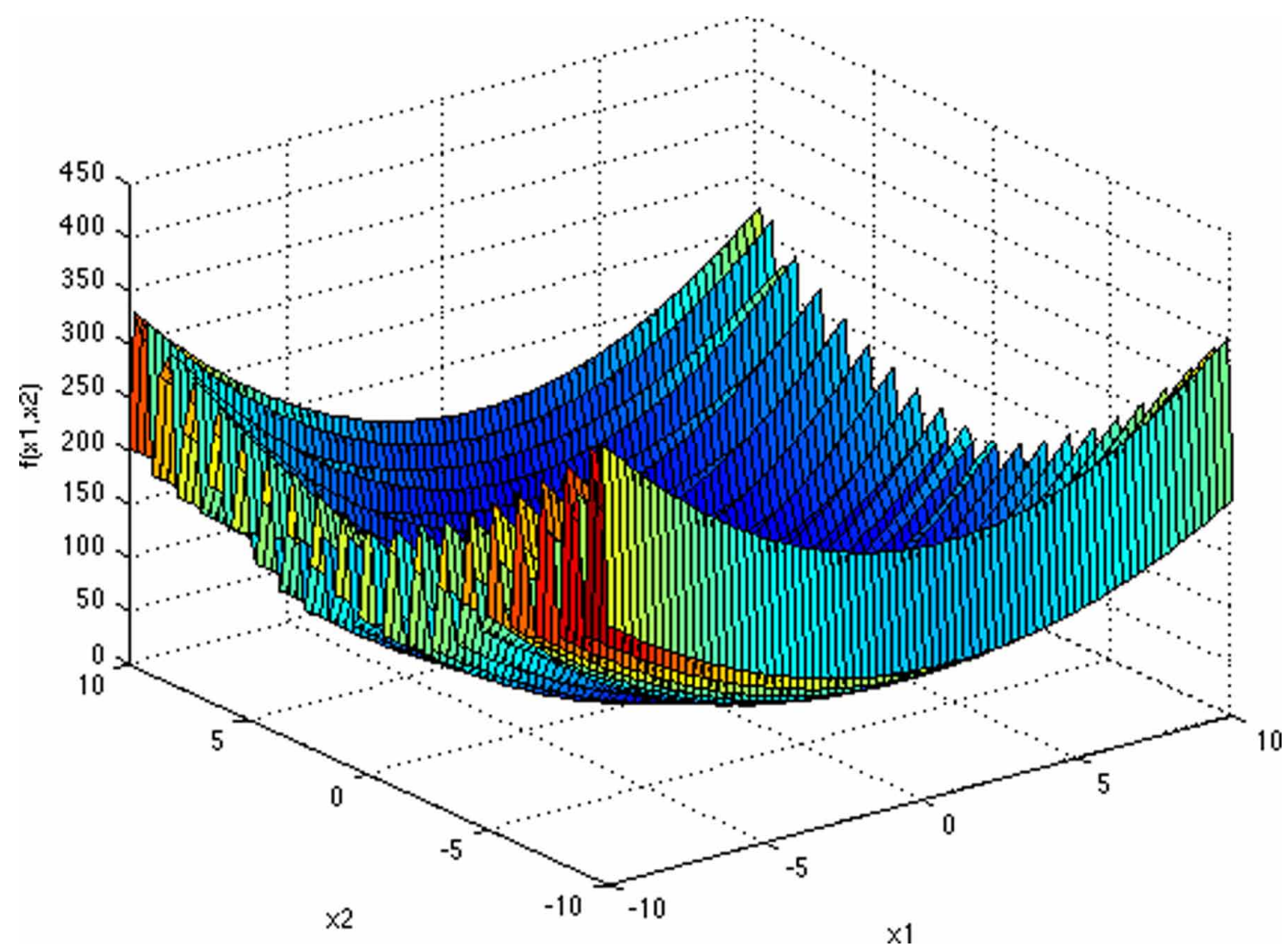

$f(y)=-\cos \left(y_{1}\right) \cos \left(y_{2}\right) \exp \left(-\left(y_{1}-\pi\right)^{2}-\left(y_{2}-\pi\right)^{2}\right)$,

\section{- Goldstein and Price (GP)}

The model is mainly estimated on the square $x_{i} \in[-2,2], \forall i=1,2$. The optimal value of this function is 3 with a single global minimum at $(0,-1)$ as shown in Figure 10. The mathematical formulation of this problem can be described as in Equation 11 (Surjanovic \& Bingham, 2013).

$$
\begin{aligned}
& f(y)=\left[1+\left(y_{1}+y_{2}+1\right)^{2}\left(19-14 y_{1}+3 y_{1}^{2}-14 y_{2}+6 y_{1} y_{2}+3 y_{2}^{2}\right)\right] \times \\
& {\left[30+\left(2 y_{1}-3 y_{2}\right)^{2}\left(18-32 y_{1}+12 y_{1}^{2}+48 y_{2}-36 y_{1} y_{2}+27 y_{2}^{2}\right)\right],}
\end{aligned}
$$

\section{- Shubert}

The model is mainly evaluated on the square $x_{i} \in[-10,10], \forall i=1,2$, although this may be restricted to the square $x_{i} \in[-5.12,5.12], \forall i=1,2$. The optimal value of this benchmark is -186.7309 , as shown in Figure 11, which the zoomed-in plot presents its multiple local minima. The mathematical formulation of this problem can be described as in Equation 12 (Surjanovic \& Bingham, 2013). 
Figure 8. Branin function

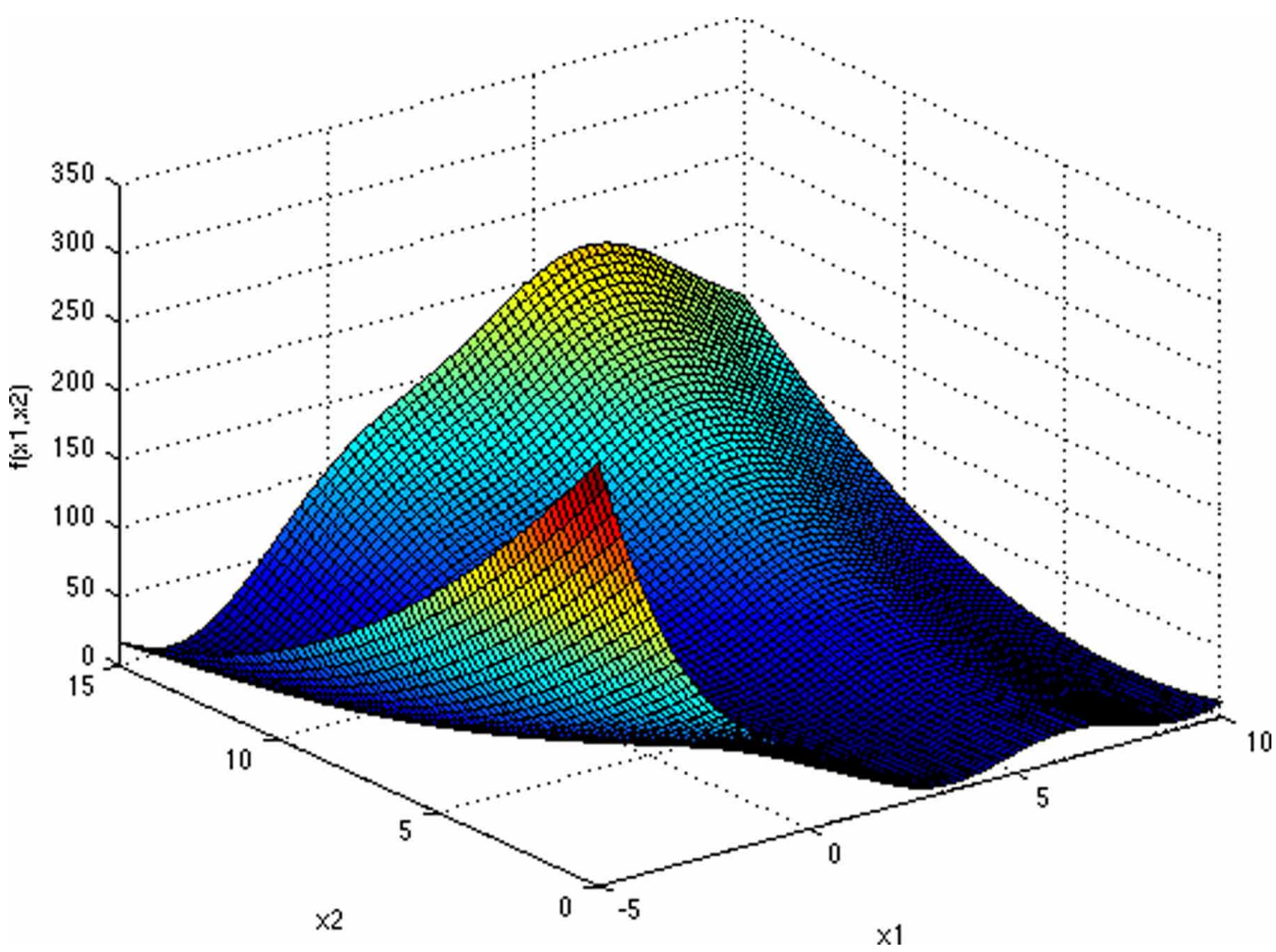

$f(y)=\left(\sum_{i=1}^{5} i \cos \left((i+1) y_{1}+i\right)\right)\left(\sum_{i=1}^{5} i \cos \left((i i+1) y_{2}+i\right)\right)$

\section{- Shekel}

Shekel function is usually evaluated on 4 dimensions search space domain, which has multiple local minima and 4 global minima values. This benchmark is mainly evaluated on the hypercube $x_{i}$ $\in[0,10], \forall i=1,2,3,4$. The optimal value of this function is mainly less or equal to -10.5 in which is changed depends on the value of $m$ and $x$. The mathematical formulation of this problem can be represented as in Equation 13 (Surjanovic \& Bingham, 2013).

$$
f(y)=\sum_{i=1}^{m}\left(\sum_{j=1}^{4}\left(y_{j}-c_{j i}\right)^{2}+\beta_{i}\right)^{-1}
$$

Where, 


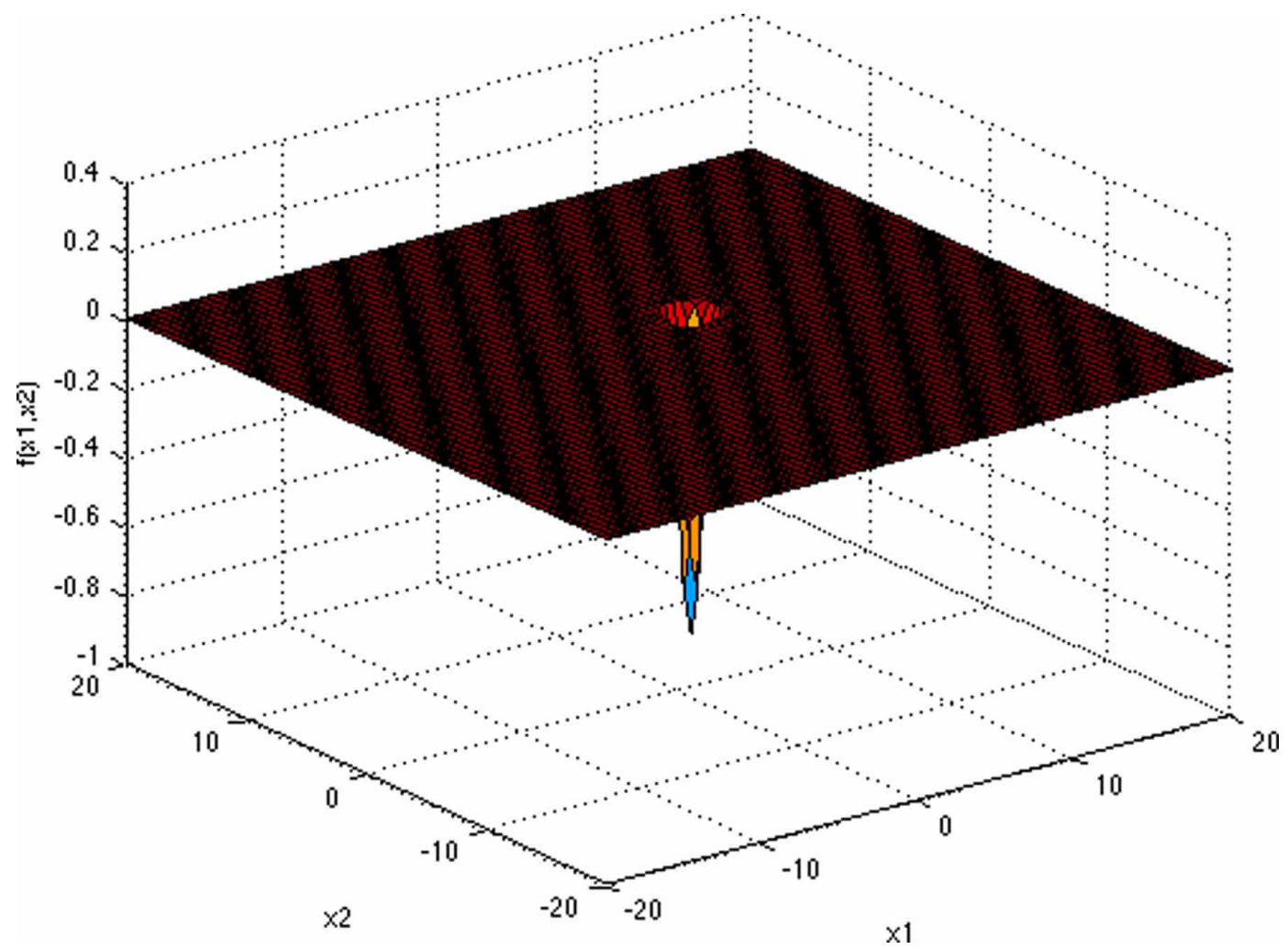

$\beta=\frac{1}{10}(1,2,2,4,4,6,3,7,5,5)^{\mathrm{T}}$,

$c=\left(\begin{array}{lllllllllll}4.0 & 1.0 & 8.0 & 6.0 & 3.0 & 2.0 & 5.0 & 8.0 & 6.0 & 7.0 \\ 4.0 & 1.0 & 8.0 & 6.0 & 7.0 & 9.0 & 3.0 & 1.0 & 2.0 & 3.6 \\ 4.0 & 1.0 & 8.0 & 6.0 & 3.0 & 2.0 & 5.0 & 8.0 & 6.0 & 7.0 \\ 4.0 & 1.0 & 8.0 & 6.0 & 7.0 & 9.0 & 3.0 & 1.0 & 2.0 & 3.6\end{array}\right)$,

The MatLab 2017a has been utilized to implement the proposed approach. All of the tests are carried out on a computer with 4 GB RAM and Intel dual-core CPU T3200 running Windows 7. The parameters of all the algorithms have been standardized during the evaluation of the proposed benchmark problems, as recommended in (Shehadeh et al., 2018b; Shehadeh et al., 2018c; Saravanan $\&$ Sachithanandam, 2001) to compare the result of HGASSO against the results of GA and SSO. The parameters of HGASSO, SSO, and GA are listed in Table 1, which the parameters of SSO are set as follows; $D$ is a velocity damping factor, which sets in the range of $(0,1) ; p H \_R a n d_{1}, p H \_R a n d_{2}$, and pH_Rand 3 are the potential of Hydrogen values of the visited location, which set in the range of (7, 14). Temp_Rand 1 and Temp_Rand ${ }_{2}$ are the temperature parameters of the visited location, which set in the range of $(35.1,38.5)$ (Shehadeh et al., 2018b; Shehadeh et al., 2018c). The parameters of GA 
Figure 10. Goldstein and Price (GP) function

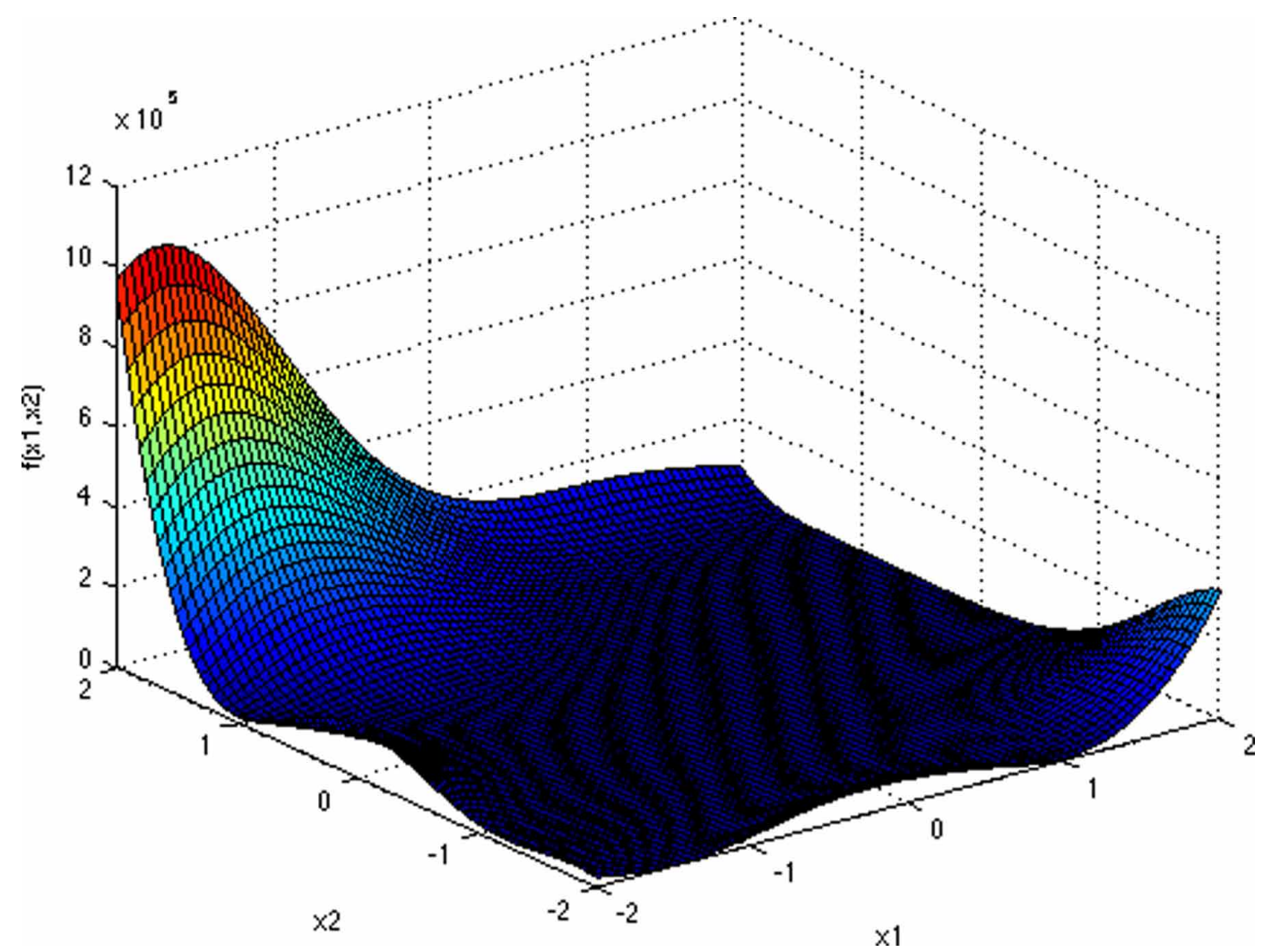

Figure 11. Shubert function
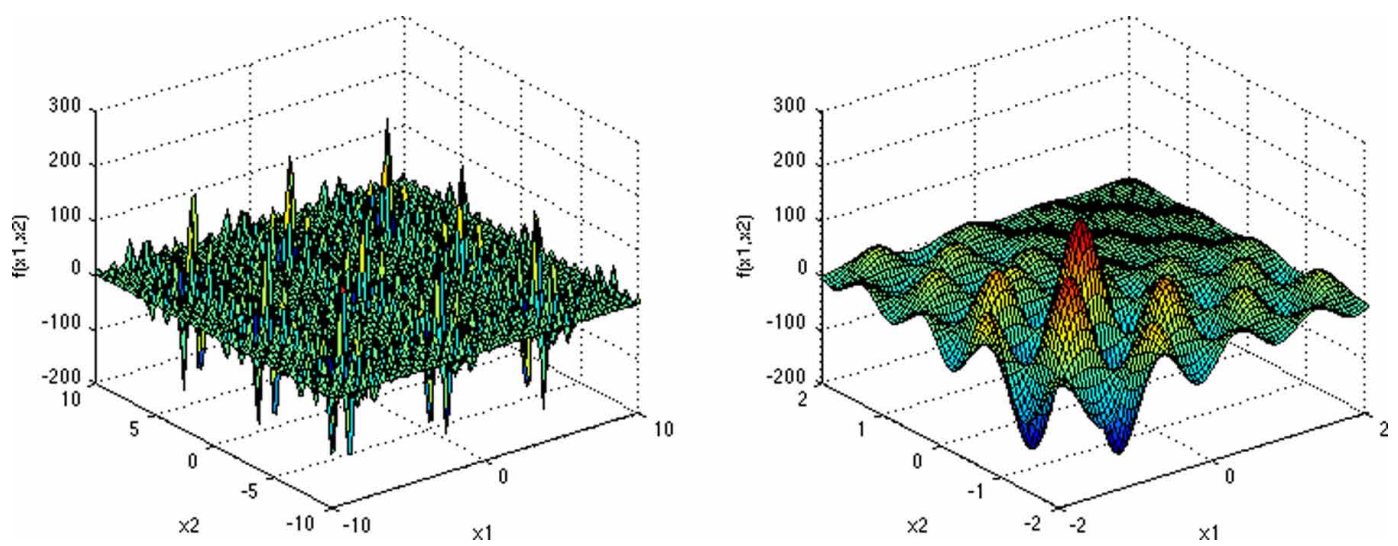

are set as follows; crossover and mutation are set to 0.8 and 0.05 respectively as in (Shehadeh et al., 2018c; Saravanan \& Sachithanandam, 2001).

Overall, every test function is evaluated in a total of ten-time runs. The experimental outcomes for HGASSO, SSO, and GA are presented in Tables from 2 to 12. Each table shows the number of iterations, the best fitness value of each benchmark problem, the average value, median value, maximum value, and the standard deviation. The algorithm convergences of HGASSO, SSO, and GA 
are depicted in Figures from 12 to 22. We follow the standard strategy of comparing the proposed approach against GA and SSO in which the number of generations has been changed through the evaluation. This scenario of evolution is used to estimate many well-known approaches, such as in (Shehadeh et al., 2018c; Banharnsakun et al, 2011; Sathya \& Radhika, 2013; Rbouh \& Imrani, 2014; Shi \& Eberhart, 1999).

Overall, the proposed HGASSO algorithm obtained the best fitness for Rastrigin, Ackley, Bukin, Griewank, Levy function N.13, and Shekel benchmark functions, followed by SSO and GA as outlined in Table 13. However, for Easom and Shubert test functions, the proposed HGASSO attained the same rank with SSO. In addition, for Cross-in-Tray, Branin, and Goldstein and Price (GP) test functions, the proposed HGASSO attained the same rank of best fitness value with SSO and GA. For the average best of ten-runs on the proposed benchmarks test functions, the HGASSO is the best on Rastrigin, Ackley, Bukin, Griewank, Levy function N.13, Branin, Easom (ES), Goldstein and Price (GP), and Shubert test functions. However, for Cross-in-Tray and Shekel test functions, the proposed HGASSO attained the same rank of best average with SSO. This shows that the proposed HGASSO has a better convergence than the other algorithms to explore the search space domain. Particularly, in solving the very complex benchmarks functions that contain exponent, cosine, sine, summation, and multiplications of multiple variables in their formulation. This is clear in the algorithm convergence measurement as depicted in the prior Figures from 12 to 22 in which HGASSO has the ability more than other approaches in skipping the multiple local extremes of the problems.

Table 14 presents the statistical analysis using "one-way ANOVA (Tukey's test)" for all benchmarks functions when the population size for all the approaches is set to 1000 . As outlined in the

Table 1. Parameters of the algorithms

\begin{tabular}{|c|c|}
\hline Parameters & Value \\
\hline \multicolumn{2}{|c|}{ HGASSO } \\
\hline Crossover probability & 0.8 \\
\hline Probability of mutation & 0.05 \\
\hline velocity damping factor (D) & Rand $(0,1)$ \\
\hline Temperature & Rand $(35.5,38.5)$ \\
\hline $\mathrm{pH}$ & Rand $(7,14)$ \\
\hline Population sizes & 20 \\
\hline Numbers of generations/iterations & $30,100,400$, and 1000 \\
\hline \multicolumn{2}{|c|}{ SSO } \\
\hline velocity damping factor (D) & Rand $(0,1)$ \\
\hline Temperature & Rand $(35.5,38.5)$ \\
\hline $\mathrm{pH}$ & Rand $(7,14)$ \\
\hline Population sizes & 20 \\
\hline Numbers of generations/iterations & $30,100,400$, and 1000 \\
\hline \multicolumn{2}{|c|}{ GA } \\
\hline Probability of mutation & 0.05 \\
\hline Crossover probability & 0.8 \\
\hline Population sizes & 20 \\
\hline Numbers of generations/iterations & $30,100,400$, and 1000 \\
\hline
\end{tabular}


Table 2. Comparison of experimental results between HGASSO, SSO, and GA in terms of mean, standard deviation, maximam value, median, and best fitness for the Rastrigin test function. The bold font with the highlighted background represents the best fitness and mean for the respective benchmark function

\begin{tabular}{|c|c|c|c|c|c|c|c|}
\hline Algorithm & Iteration no. & Average, mean & Std. & Max. & Median & Best fitness \\
\hline \multirow{6}{*}{ HGASSO } & 30 & 0.83069 & 2.3583 & 5.354263043 & 0.00056906 & $6.499 \mathrm{e}-05$ \\
\cline { 2 - 7 } & 100 & 0.02177 & 0.38374 & 1.22085478 & $9.77392 \mathrm{e}-07$ & $9.7739 \mathrm{e}-07$ \\
\cline { 2 - 7 } & 400 & 0.018452 & 0.24184 & 4.345719212 & $1.42109 \mathrm{e}-14$ & $1.4211 \mathrm{e}-14$ \\
\cline { 2 - 8 } & 1000 & 0.046841 & 0.27594 & 4.191540893 & 0 & 0 \\
\hline \multirow{4}{*}{ SSO } & 30 & 1.058 & 0.224 & 2.0155 & 0.99520 & 0.99496 \\
\cline { 2 - 8 } & 100 & 1.023 & 0.22607 & 3.248725968 & 0.994959057 & 0.029416 \\
\cline { 2 - 8 } & 400 & 0.074 & 0.2228 & 1.180860894 & 0.020193641 & 0.010194 \\
\cline { 2 - 8 } & 1000 & 0.053 & 0.27557 & 8.073771956 & 0 & 0 \\
\hline \multirow{6}{*}{ GA } & 30 & 7.598 & 3.4325 & 15.98928286 & 0.99589 & 0.99496 \\
\cline { 2 - 8 } & 100 & 1.6349 & 0.87803 & 5.100138537 & 1.060852 & 0.99496 \\
\cline { 2 - 7 } & 400 & 0.76901 & 1.6062 & 24.12894 & 0.087708 & 0.017129 \\
\cline { 2 - 7 } & 1000 & 0.29101 & 0.50583 & 10.4 & $6.14716 \mathrm{e}-06$ & $6.1472 \mathrm{e}-06$ \\
\hline
\end{tabular}

Figure 12. Comparison of experimental results between HGASSO, SSO, and GA in term of convergence for the Rastrigin test function
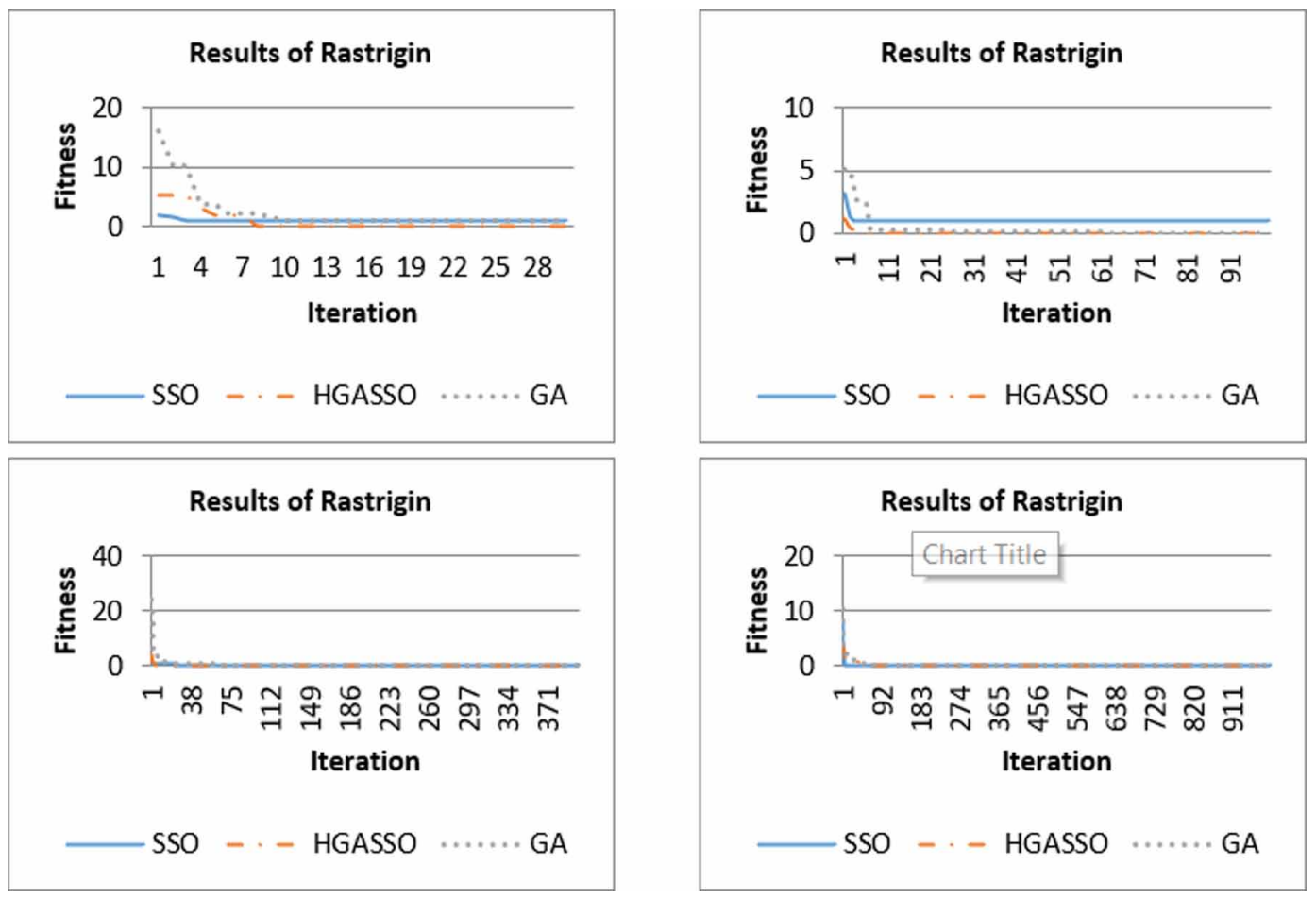
Table 3. Comparison of experimental results between HGASSO, SSO, and GA in terms of mean, standard deviation, maximam value, median, and best fitness for the Ackley test function. The bold font with the highlighted background represents the best fitness and mean for the respective benchmark function

\begin{tabular}{|c|c|c|c|c|c|c|c|}
\hline Algorithm & Iteration no. & Average, mean & Std. & Max. & Median & Best fitness \\
\hline \multirow{4}{*}{ HGASSO } & 30 & $\mathbf{0 . 3 3 7 4 3}$ & 0.99988 & 5.670564 & $0.4076 \mathrm{E}-05$ & $\mathbf{2 . 4 3 6 6 E - 0 8}$ \\
\cline { 2 - 7 } & 100 & $\mathbf{0 . 0 7 3 6}$ & 0.64297 & 7.352609216 & $0.114 \mathrm{E}-11$ & $\mathbf{8 . 0 8 2 4 E - 1 4}$ \\
\cline { 2 - 8 } & 400 & $\mathbf{0 . 0 1 7 3 6 2}$ & 0.2218 & 8.111862635 & $3.991 \mathrm{E}-13$ & $\mathbf{1 . 8 6 4 9 E}-15$ \\
\cline { 2 - 8 } & 1000 & $\mathbf{0 . 0 0 5 4 6 7 8}$ & 0.12183 & 6.923460765 & $6.91 \mathrm{E}-16$ & $\mathbf{6 . 9 1 6 5 E}-16$ \\
\hline \multirow{4}{*}{ SSO } & 30 & 0.45821 & 0.99405 & 5.516434 & 0.001582 & $2.9937 \mathrm{E}-08$ \\
\cline { 2 - 8 } & 100 & 0.18659 & 0.50742 & 7.669341 & $1.52 \mathrm{E}-11$ & $9.8412 \mathrm{E}-14$ \\
\cline { 2 - 8 } & 400 & 0.023298 & 0.24924 & 7.347074 & $8.8 \mathrm{E}-15$ & $2.6645 \mathrm{E}-15$ \\
\cline { 2 - 8 } & 1000 & 0.0078659 & 0.15351 & 10.4051 & $8.88 \mathrm{E}-16$ & $8.8818 \mathrm{E}-16$ \\
\hline \multirow{6}{*}{ GA } & 30 & 3.1013 & 1.9973 & 6.623962 & 2.34069 & 1.7499 \\
\cline { 2 - 8 } & 100 & 1.951 & 1.2934 & 6.162792 & 1.6357 & 1.2311 \\
\cline { 2 - 8 } & 400 & 1.4138 & 0.76135 & 3.379967 & 1.5219 & 1.2312 \\
\cline { 2 - 8 } & 1000 & 1.7328 & 0.44703 & 3.332738 & 1.8725 & 1.6505 \\
\hline
\end{tabular}

Figure 13. Comparison of experimental results between HGASSO, SSO, and GA in term of convergence for the Ackley test function
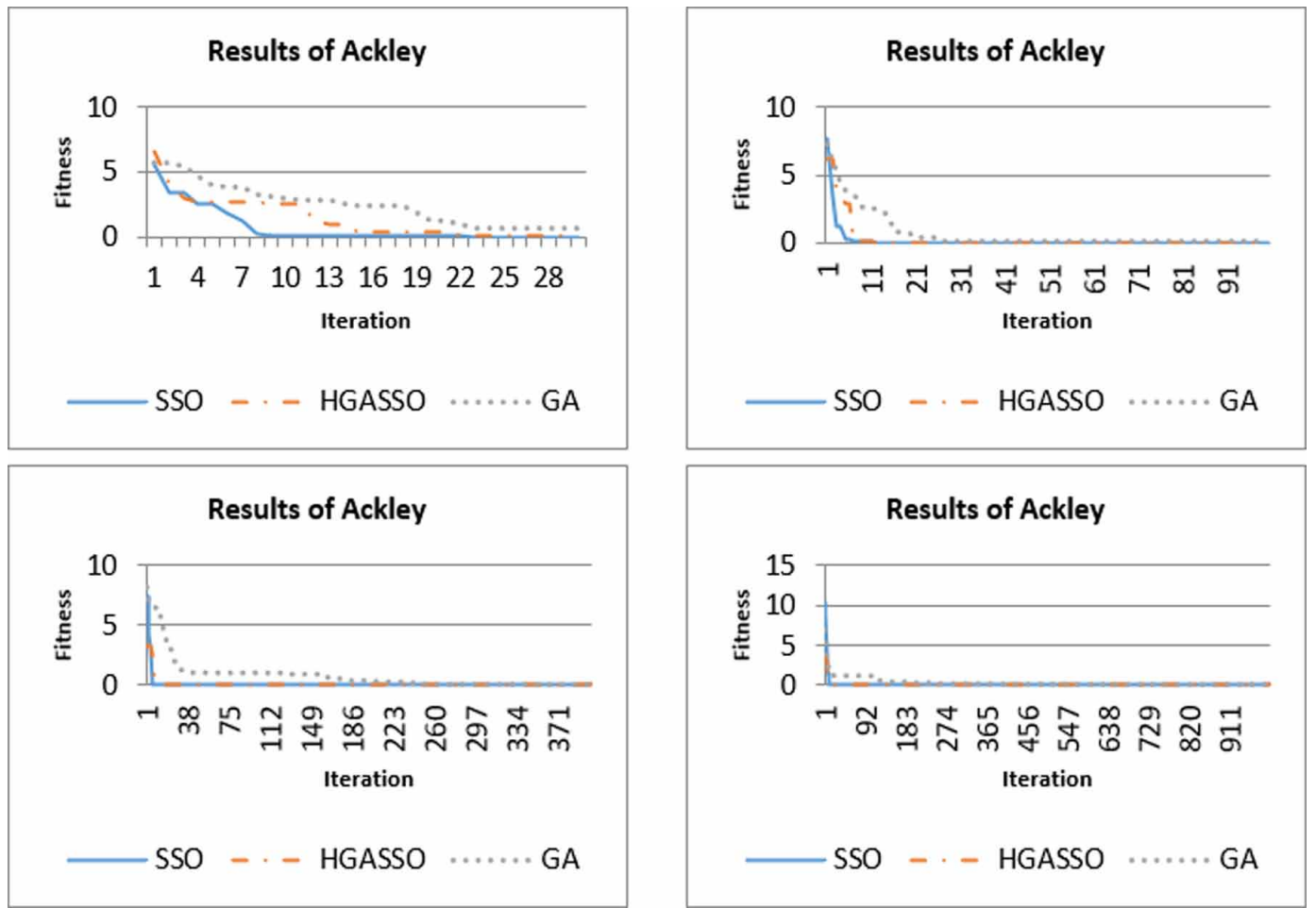
Table 4. Comparison of experimental results between HGASSO, SSO, and GA in terms of mean, standard deviation, maximam value, median, and best fitness for the Bukin test function. The bold font with the highlighted background represents the best fitness and mean for the respective benchmark function

\begin{tabular}{|c|c|c|c|c|c|c|c|}
\hline Algorithm & Iteration no. & Average, mean & Std. & Max. & Median & Best fitness \\
\hline \multirow{6}{*}{ HGASSO } & 30 & $\mathbf{3 . 1 5 3 8}$ & 9.4634 & 32.4804 & 0.09003 & $\mathbf{0 . 0 4 6 9 1}$ \\
\cline { 2 - 7 } & 100 & $\mathbf{0 . 4 9 0 6}$ & 2.306 & 14.23453 & 0.065994 & $\mathbf{0 . 0 6 5 9 9 4}$ \\
\cline { 2 - 7 } & 400 & $\mathbf{0 . 1 4 7 8 3}$ & 0.9582 & 12.58949 & 0.0507 & $\mathbf{0 . 0 4 4 7 7 7 7}$ \\
\cline { 2 - 7 } & 1000 & $\mathbf{0 . 1 0 5 1 3}$ & 0.6923 & 16.2696 & 0.053808 & $\mathbf{0 . 0 5 3 8 0 8}$ \\
\hline \multirow{6}{*}{ SSO } & 30 & 34.488 & 7.9943 & 38.08249 & 0.431059 & 0.09003 \\
\cline { 2 - 7 } & 100 & 5.6346 & 2.1636 & 16.89265 & 0.126685 & 0.12669 \\
\cline { 2 - 7 } & 400 & 1.9536 & 0.67845 & 122.5681 & 0.044777 & 0.0447 \\
\cline { 2 - 7 } & 1000 & 0.5872 & 0.73588 & 16.41006 & 0.055188 & 0.055188 \\
\hline \multirow{6}{*}{ GA } & 30 & 13.5755 & 9.1456 & 31.43089 & 0.838493 & 0.199 \\
\cline { 2 - 7 } & 100 & 8.6237 & 11.7059 & 74.92827 & 0.237401 & 0.13858 \\
\cline { 2 - 7 } & 400 & 1.8721 & 3.1999 & 42.25904 & 0.058669 & 0.058669 \\
\cline { 2 - 7 } & 1000 & 0.54051 & 3.1527 & 86.18405 & 0.140932 & 0.0714093 \\
\hline
\end{tabular}

Figure 14. Comparison of experimental results between HGASSO, SSO, and GA in term of convergence for the Bukin test function
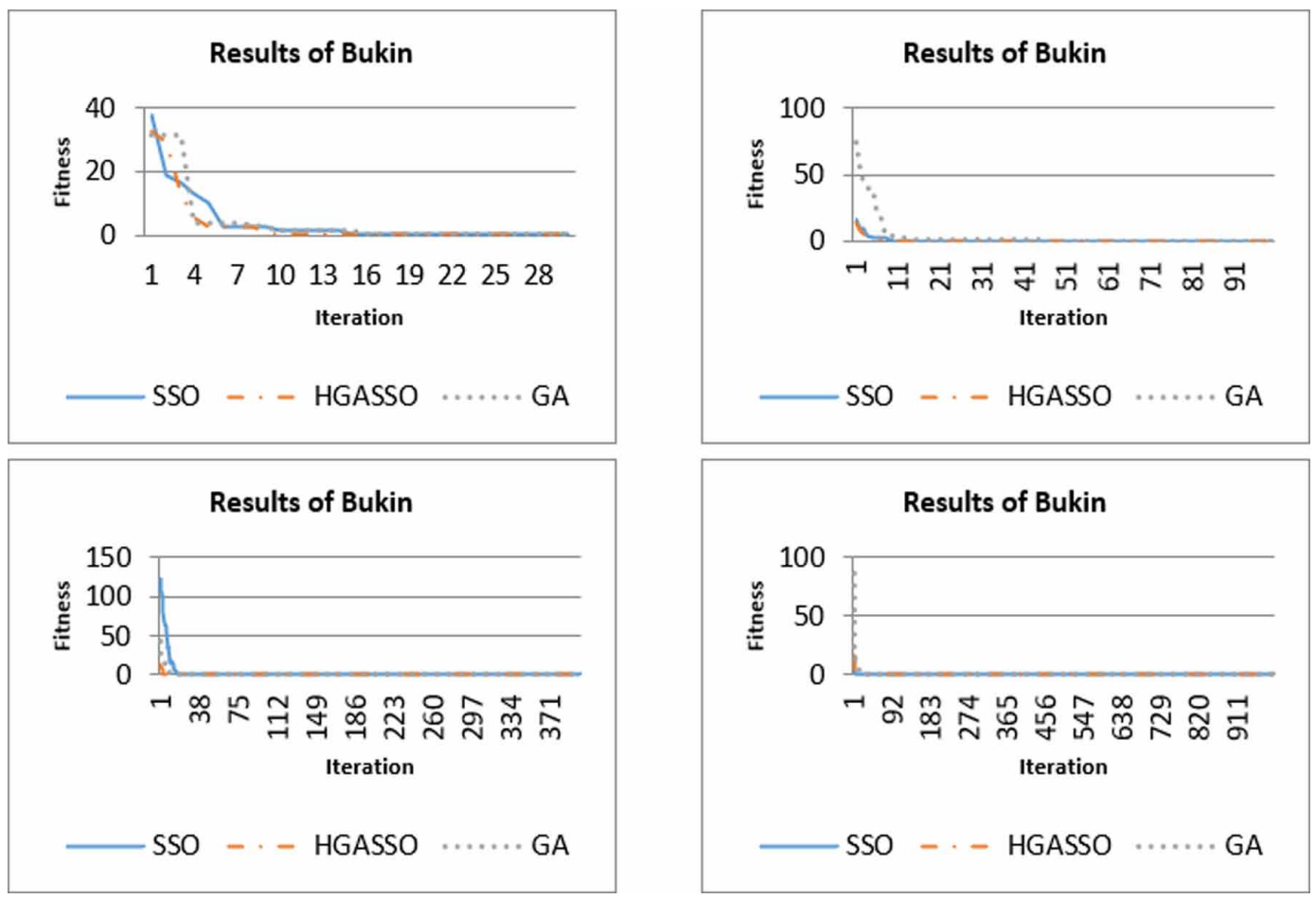
Table 5. Comparison of experimental results between HGASSO, SSO, and GA in terms of mean, standard deviation, maximam value, median, and best fitness for the Griewank test function. The bold font with the highlighted background represents the best fitness and mean for the respective benchmark function

\begin{tabular}{|c|c|c|c|c|c|c|c|}
\hline Algorithm & Iteration no. & Average, mean & Std. & Max. & Median & Best fitness \\
\hline \multirow{6}{*}{ HGASSO } & 30 & $\mathbf{0 . 0 7 2 4 7 7 2}$ & 0.016421 & 0.025229 & $6.31 \mathrm{E}-05$ & $\mathbf{6 . 2 7 5 1 e - 0 5}$ \\
\cline { 2 - 7 } & 100 & $\mathbf{0 . 0 1 2 3 6 6}$ & 0.0080656 & 0.039221 & 0.007408 & $\mathbf{0 . 0 0 7 3 9 1}$ \\
\cline { 2 - 7 } & 400 & $\mathbf{0 . 0 0 5 7 3 7 2}$ & 0.01106 & 0.193659 & 0.009865 & $\mathbf{2 . 1 9 5 9 e - 0 5}$ \\
\cline { 2 - 7 } & 1000 & $\mathbf{0 . 0 0 1 1 0 5 9}$ & 0.0060654 & 0.076761 & $1.558 \mathrm{E}-06$ & $\mathbf{5 . 3 5 8 4 e - 0 8}$ \\
\hline \multirow{6}{*}{ SSO } & 30 & 5.0707 & 2.366 & 12.94653 & 0.034655 & 0.01972 \\
\cline { 2 - 7 } & 100 & 2.5189 & 0.36012 & 2.60039 & 0.081556 & 0.081362 \\
\cline { 2 - 7 } & 400 & 0.75265 & 0.20696 & 3.587809 & 0.206964 & 0.20696 \\
\cline { 2 - 7 } & 1000 & 0.34638 & 0.33338 & 10.38389 & 0.13664 & 0.13664 \\
\hline \multirow{6}{*}{ GA } & 30 & 0.098954 & 0.015128 & 0.062387 & 0.013702 & 0.013702 \\
\cline { 2 - 7 } & 100 & 0.037079 & 0.0016401 & 0.016512 & 0.007396 & 0.007396 \\
\cline { 2 - 7 } & 400 & 0.012626 & 0.00048227 & 0.010224 & 0.007396 & 0.007396 \\
\cline { 2 - 7 } & 1000 & 0.010697 & 0.0054164 & 0.096065 & 0.007396 & 0.0073962 \\
\hline
\end{tabular}

Figure 15. Comparison of experimental results between HGASSO, SSO, and GA in term of convergence for the Griewank test function
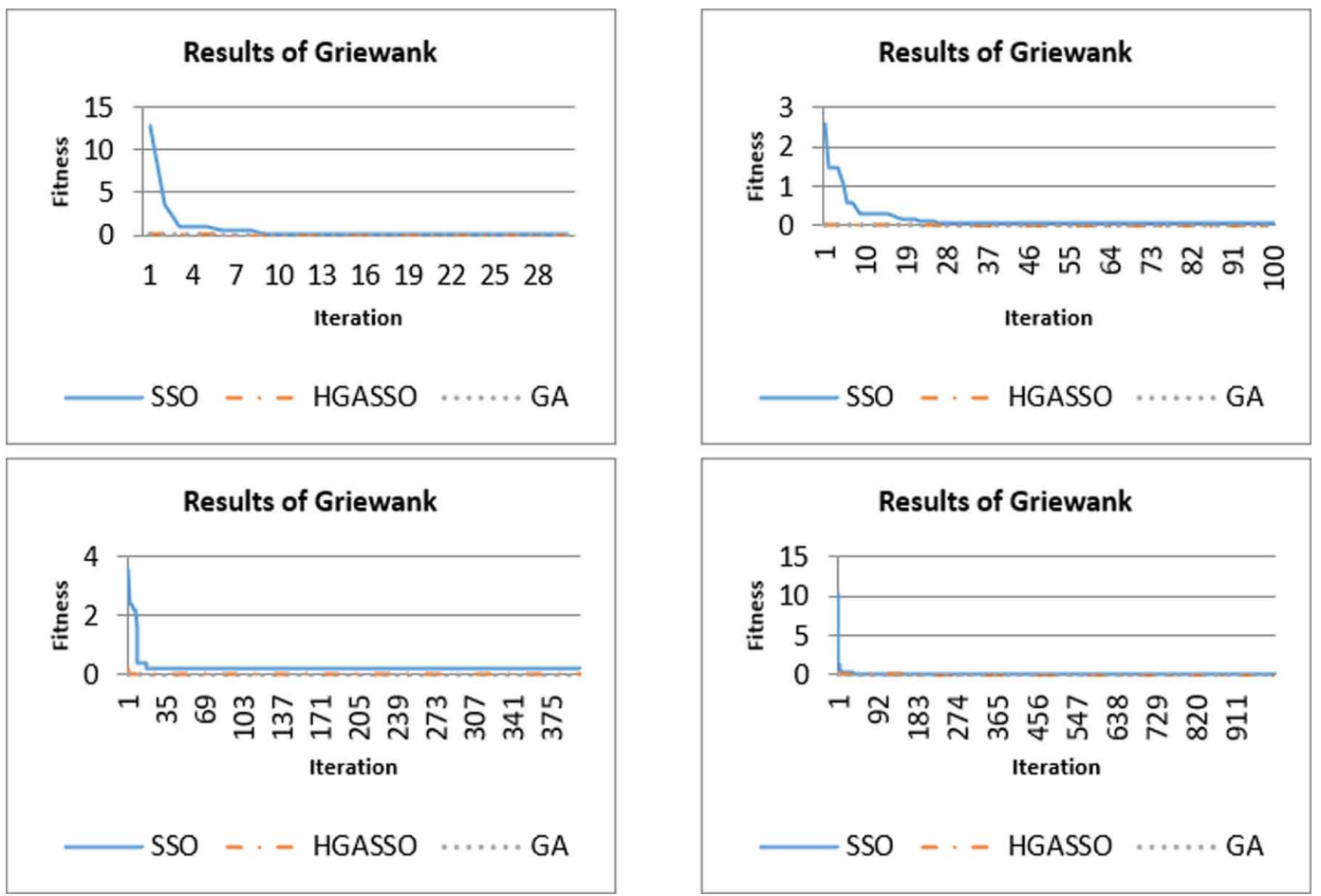
Table 6. Comparison of experimental results between HGASSO, SSO, and GA in terms of mean, standard deviation, maximam value, median, and best fitness for the Cross-in-Tray test function. The bold font with the highlighted background represents the best fitness and mean for the respective benchmark function

\begin{tabular}{|c|c|c|c|c|c|c|}
\hline Algorithm & Iteration no. & Average, mean & Std. & Max. & Median & Best fitness \\
\hline \multirow{4}{*}{ HGASSO } & 30 & $\mathbf{- 2 . 0 6 2 4}$ & 0.0065705 & -2.06036 & -2.06261 & $\mathbf{- 2 . 0 6 2 6}$ \\
\cline { 2 - 7 } & 100 & $\mathbf{- 2 . 0 6 2 6}$ & 0.0019581 & -2.06004 & -2.06261 & $\mathbf{- 2 . 0 6 2 6}$ \\
\cline { 2 - 7 } & 400 & $\mathbf{- 2 . 0 6 2 6}$ & 0.00065859 & -2.05285 & -2.06261 & $\mathbf{- 2 . 0 6 2 6}$ \\
\cline { 2 - 7 } & 1000 & $\mathbf{- 2 . 0 6 2 6}$ & 0.001813 & -2.006 & -2.06261 & $\mathbf{- 2 . 0 6 2 6}$ \\
\hline \multirow{4}{*}{ SSO } & 30 & -1.9725 & 0.061039 & -1.86304 & -2.06261 & $\mathbf{- 2 . 0 6 2 6}$ \\
\cline { 2 - 7 } & 100 & -2.0314 & 0.0017321 & -2.05025 & -2.06261 & $\mathbf{- 2 . 0 6 2 6}$ \\
\cline { 2 - 7 } & 400 & $\mathbf{- 2 . 0 6 2 6}$ & 0.0014933 & -2.04194 & -2.06261 & $\mathbf{- 2 . 0 6 2 6}$ \\
\cline { 2 - 7 } & 1000 & $\mathbf{- 2 . 0 6 2 6}$ & 0.0020119 & -2.00025 & -2.06261 & $\mathbf{- 2 . 0 6 2 6}$ \\
\hline \multirow{6}{*}{ GA } & 30 & -2.0324 & 0.011546 & -2.01877 & -2.06261 & $\mathbf{- 2 . 0 6 2 6}$ \\
\cline { 2 - 7 } & 100 & -2.0547 & 0.00077371 & -2.05743 & -2.06261 & $\mathbf{- 2 . 0 6 2 6}$ \\
\cline { 2 - 7 } & 400 & -2.0601 & 0.0036624 & -2.03175 & -2.06261 & $\mathbf{- 2 . 0 6 2 6}$ \\
\hline
\end{tabular}

Figure 16. Comparison of experimental results between HGASSO, SSO, and GA in term of convergence for the Cross-in-Tray test function
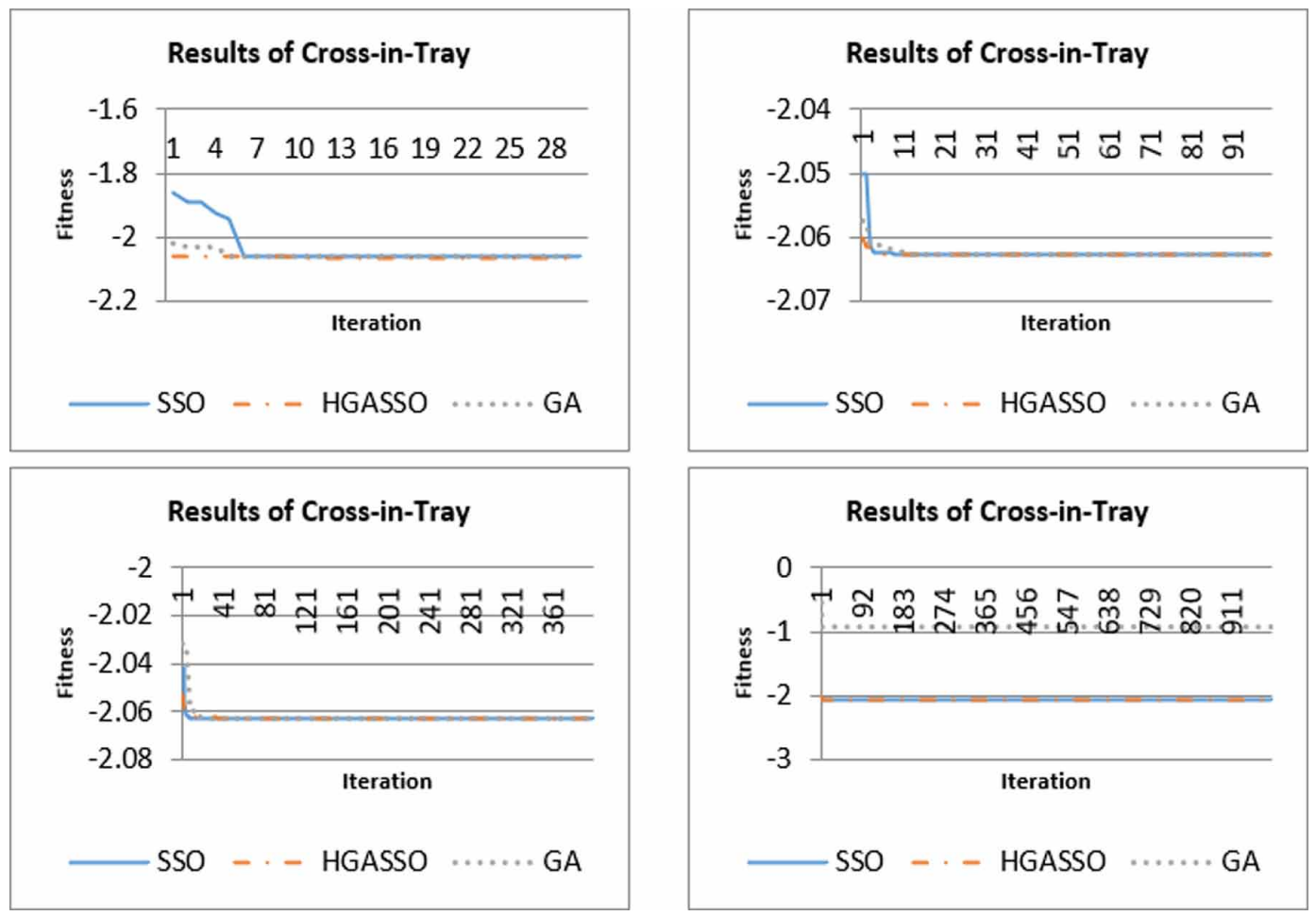
Table 7. Comparison of experimental results between HGASSO, SSO, and GA in terms of mean, standard deviation, maximam value, median, and best fitness for the Levy N.13 test function. The bold font with the highlighted background represents the best fitness and mean for the respective benchmark function

\begin{tabular}{|c|c|c|c|c|c|c|c|}
\hline Algorithm & Iteration no. & Average, mean & Std. & Max. & Median & Best fitness \\
\hline \multirow{6}{*}{ HGASSO } & 30 & $\mathbf{0 . 1 1 2 6 3}$ & 0.48914 & 2.112283 & $1.67 \mathrm{E}-06$ & $\mathbf{1 . 0 0 5 4 e - 0 6}$ \\
\cline { 2 - 7 } & 100 & $\mathbf{0 . 0 0 9 8 2 0 8}$ & 0.1288 & 0.53653 & $7.18 \mathrm{E}-16$ & $\mathbf{1 . 3 4 9 8 e - 3 1}$ \\
\cline { 2 - 7 } & 400 & $\mathbf{0 . 0 0 4 0 2 9 3}$ & 0.060411 & 0.984788 & $2.21 \mathrm{E}-15$ & $\mathbf{1 . 3 4 9 8 e - 3 1}$ \\
\cline { 2 - 7 } & 1000 & $\mathbf{0 . 0 0 0 4 0 9 4 5}$ & 0.011655 & 0.213259 & $1.35 \mathrm{E}-31$ & $\mathbf{1 . 3 4 9 8 e - 3 1}$ \\
\hline \multirow{4}{*}{ SSO } & 30 & 1.0284 & 0.14993 & 1.453477 & 0.01309 & 0.0056401 \\
\cline { 2 - 7 } & 100 & 0.40176 & 0.16547 & 1.148953 & $9.39 \mathrm{E}-23$ & $1.335 \mathrm{E}-31$ \\
\cline { 2 - 7 } & 400 & 0.20985 & 0.043645 & 1.297072 & $1.349 \mathrm{E}-31$ & $1.335 \mathrm{E}-31$ \\
\cline { 2 - 7 } & 1000 & 1.0284 & 0.14993 & 1.092091 & $2.034 \mathrm{E}-30$ & $2.0345 \mathrm{e}-30$ \\
\hline \multirow{6}{*}{ GA } & 30 & 2.9547 & 1.5963 & 8.523651 & 0.014209 & $1.955 \mathrm{e}-05$ \\
\cline { 2 - 7 } & 100 & 0.88172 & 0.36938 & 2.160539 & $3.98 \mathrm{E}-11$ & $3.9844 \mathrm{e}-11$ \\
\cline { 2 - 7 } & 400 & 0.31151 & 0.37344 & 7.442983 & $4.47 \mathrm{E}-10$ & $4.4668 \mathrm{e}-13$ \\
\cline { 2 - 7 } & 1000 & 0.099797 & 0.37253 & 8.727115 & $1.35 \mathrm{E}-31$ & $1.3490 \mathrm{e}-31$ \\
\hline
\end{tabular}

Figure 17. Comparison of experimental results between HGASSO, SSO, and GA in term of convergence for the Levy N.13 test function
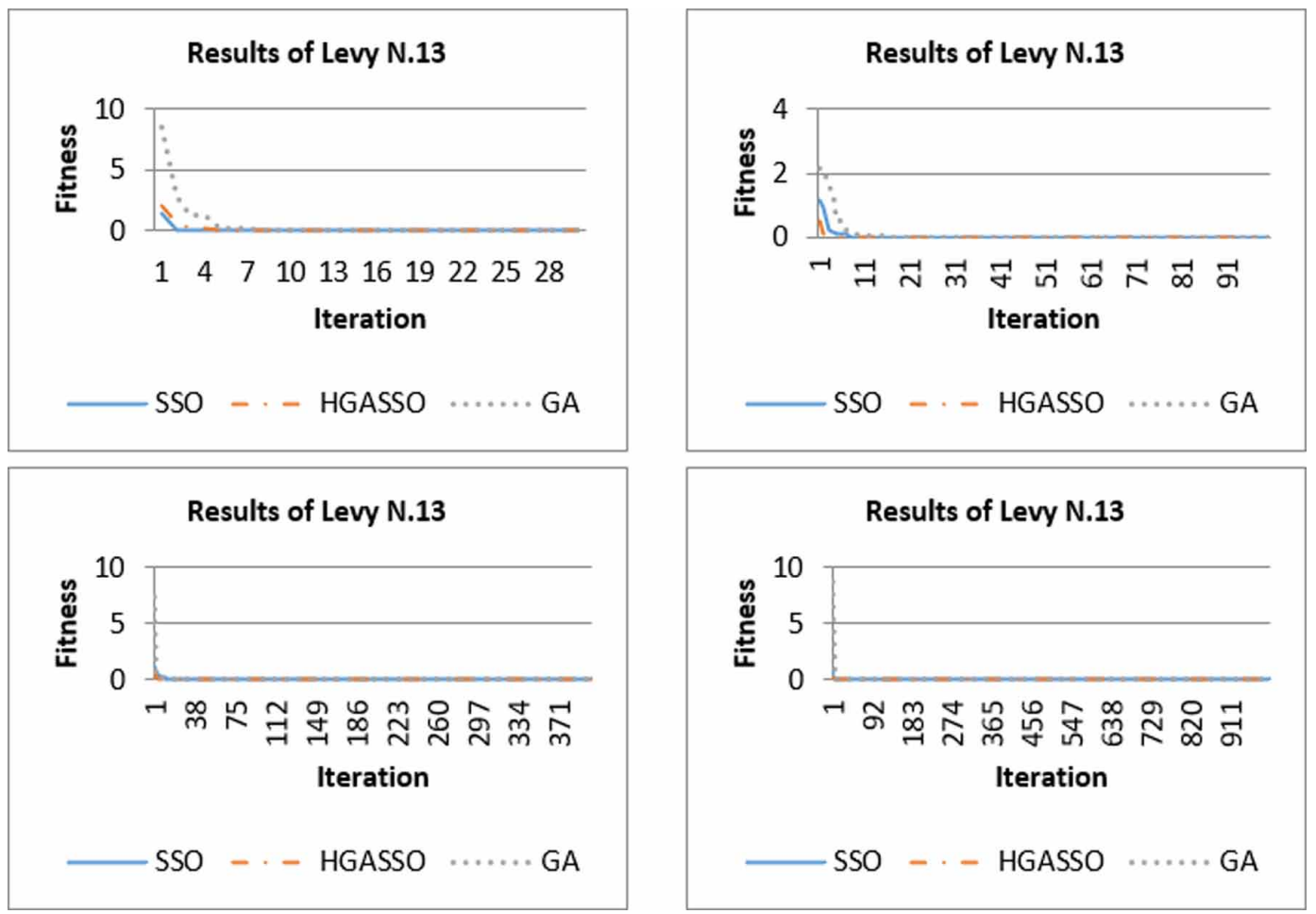
Table 8. Comparison of experimental results between HGASSO, SSO, and GA in terms of mean, standard deviation, maximam value, median, and best fitness for the Branin test function. The bold font with the highlighted background represents the best fitness and mean for the respective benchmark function

\begin{tabular}{|c|c|c|c|c|c|c|c|}
\hline Algorithm & Iteration no. & Average, mean & Std. & Max. & Median & Best fitness \\
\hline \multirow{4}{*}{ HGASSO } & 30 & $\mathbf{0 . 4 9 0 6 5}$ & 0.53949 & 2.479416 & 0.397887 & $\mathbf{0 . 3 9 7 8 9}$ \\
\cline { 2 - 7 } & 100 & $\mathbf{0 . 4 0 0 3 4}$ & 0.085994 & 0.521464 & 0.397887 & $\mathbf{0 . 3 9 7 8 9}$ \\
\cline { 2 - 7 } & 400 & $\mathbf{0 . 3 9 9 6 4}$ & 0.037387 & 0.748338 & 0.397887 & $\mathbf{0 . 3 9 7 8 9}$ \\
\cline { 2 - 7 } & 1000 & $\mathbf{0 . 3 9 8 8 4}$ & 0.031369 & 1.321689 & 0.397887 & $\mathbf{0 . 3 9 7 8 9}$ \\
\hline \multirow{6}{*}{ SSO } & 30 & 1.4434 & 0.093386 & 0.807697 & 0.397887 & $\mathbf{0 . 3 9 7 8 9}$ \\
\cline { 2 - 7 } & 100 & 1.1274 & 0.020624 & 0.605062 & 0.397887 & $\mathbf{0 . 3 9 7 8 9}$ \\
\cline { 2 - 7 } & 400 & 0.6506 & 0.030288 & 0.80531 & 0.397887 & $\mathbf{0 . 3 9 7 8 9}$ \\
\cline { 2 - 7 } & 1000 & 0.4296 & 0.030804 & 1.221217 & 0.397887 & $\mathbf{0 . 3 9 7 8 9}$ \\
\hline \multirow{6}{*}{ GA } & 30 & 6.0451 & 0.46534 & 1.912022 & 0.415508 & $\mathbf{0 . 3 9 7 8 9}$ \\
\cline { 2 - 7 } & 100 & 1.9106 & 0.17979 & 1.233101 & 0.397887 & $\mathbf{0 . 3 9 7 8 9}$ \\
\cline { 2 - 7 } & 400 & 1.2753 & 0.56752 & 9.194571 & 0.397887 & $\mathbf{0 . 3 9 7 8 9}$ \\
\hline
\end{tabular}

Figure 18. Comparison of experimental results between HGASSO, SSO, and GA in term of convergence for the Branin test function
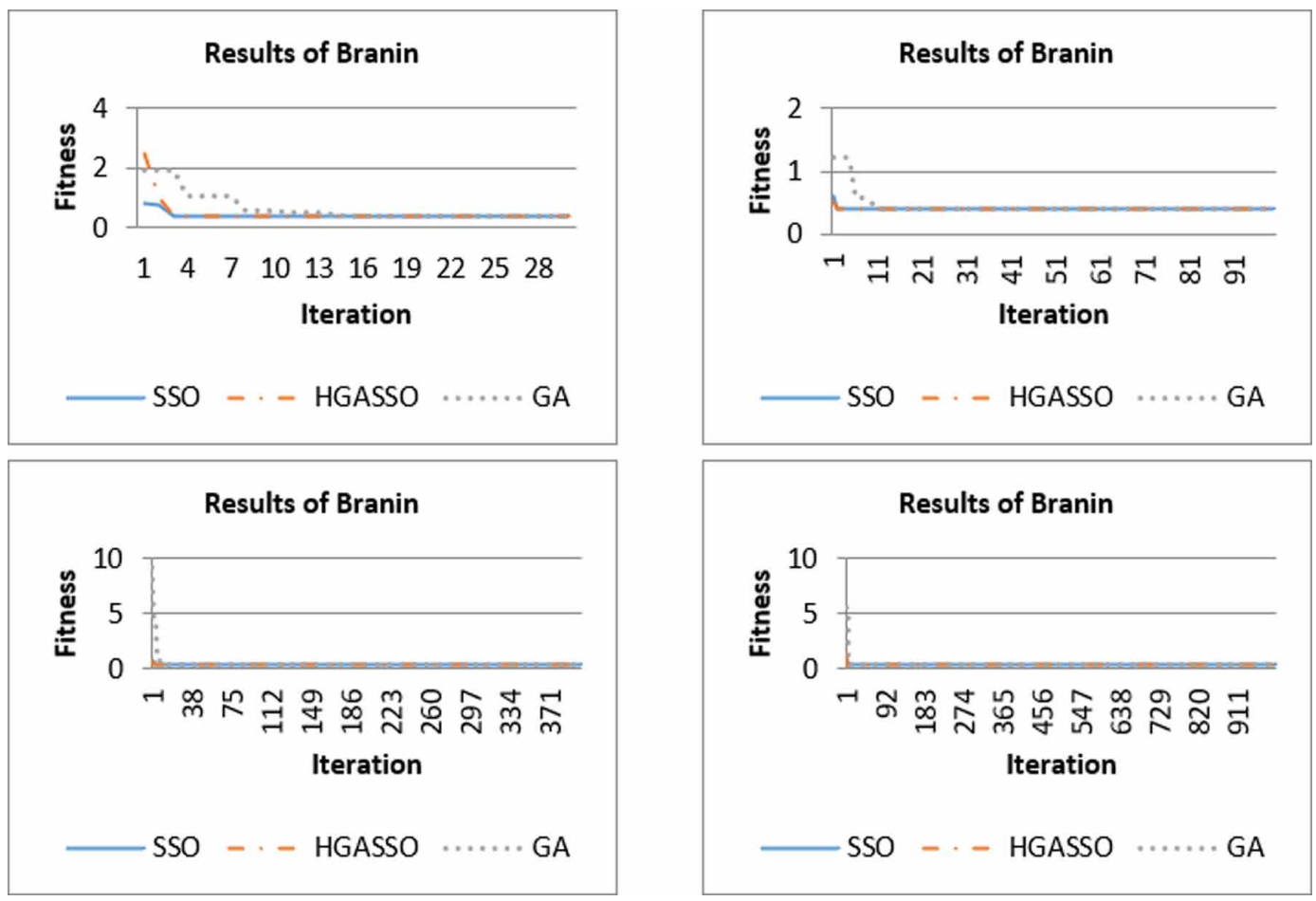
Table 9. Comparison of experimental results between HGASSO, SSO, and GA in terms of mean, standard deviation, maximam value, median, and best fitness for the Easom (ES) test function. The bold font with the highlighted background represents the best fitness and mean for the respective benchmark function

\begin{tabular}{|c|c|c|c|c|c|c|}
\hline Algorithm & Iteration no. & Average, mean & Std. & Max. & Median & Best fitness \\
\hline \multirow{6}{*}{ HGASSO } & 30 & $\mathbf{- 0 . 9 3 6 2 9}$ & 0.067942 & -0.57854 & -1 & $\mathbf{- 1}$ \\
\cline { 2 - 7 } & 100 & $\mathbf{- 0 . 9 8 9 3 6}$ & 0.19349 & -0.00023 & -1 & $\mathbf{- 1}$ \\
\cline { 2 - 7 } & 400 & $\mathbf{- 0 . 9 9 5 7 4}$ & 0.061802 & -0.00932 & -1 & $\mathbf{- 1}$ \\
\cline { 2 - 7 } & 1000 & $\mathbf{- 0 . 9 9 9 6}$ & 0.013206 & -0.58761 & -1 & $\mathbf{- 1}$ \\
\hline \multirow{4}{*}{ SSO } & 30 & -0.45032 & 0.45255 & $-1.30 \mathrm{E}-80$ & $-9.96 \mathrm{E}-01$ & $\mathbf{- 1}$ \\
\cline { 2 - 7 } & 100 & -0.78718 & 0.34859 & $-3.0 \mathrm{E}-171$ & -1 & $\mathbf{- 1}$ \\
\cline { 2 - 7 } & 400 & -0.96241 & 0.14033 & $-6.55 \mathrm{E}-84$ & -1 & $\mathbf{- 1}$ \\
\cline { 2 - 7 } & 1000 & -0.98494 & 0.080968 & 0 & -1 & $-\mathbf{1}$ \\
\hline \multirow{6}{*}{ GA } & 30 & $-2.0138 \mathrm{e}-05$ & $2.6482 \mathrm{e}-05$ & $-4.38 \mathrm{E}-07$ & $-1.24 \mathrm{E}-05$ & $-8.1095 \mathrm{e}-05$ \\
\cline { 2 - 7 } & 100 & $-7.1524 \mathrm{e}-05$ & 0.086161 & -0.70954 & -0.99288 & $-8.1101 \mathrm{e}-05$ \\
\cline { 2 - 7 } & 400 & $-7.9285 \mathrm{e}-05$ & $2.2619 \mathrm{e}-05$ & $-3.68 \mathrm{E}-13$ & $-8.11 \mathrm{E}-05$ & $-8.11 \mathrm{e}-05$ \\
\cline { 2 - 7 } & 1000 & -0.93195 & $9.2674 \mathrm{e}-06$ & $-1.12 \mathrm{E}-07$ & $-8.11 \mathrm{E}-05$ & -0.99291 \\
\hline
\end{tabular}

Figure 19. Comparison of experimental results between HGASSO, SSO, and GA in term of convergence for the Easom (ES) test function
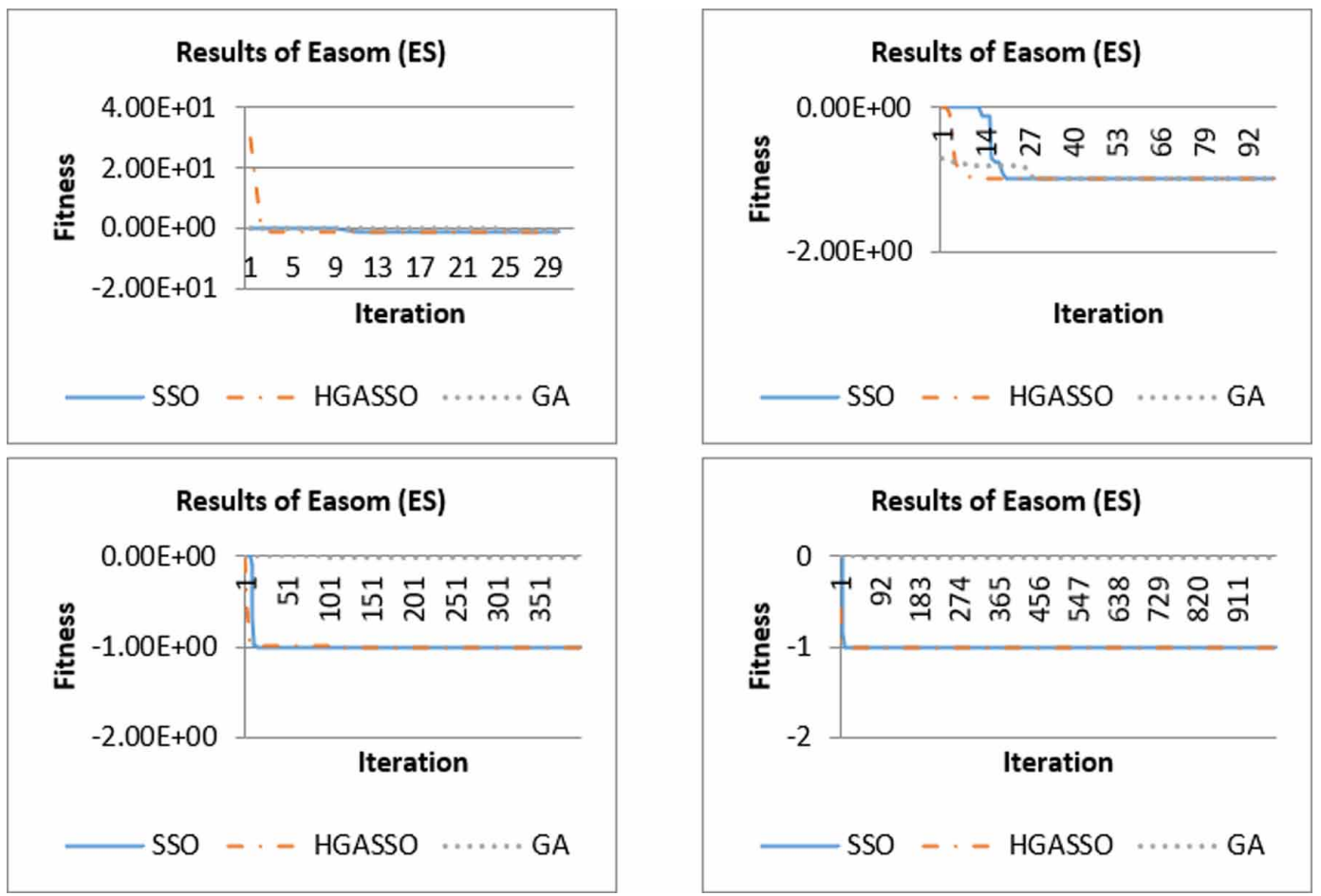
Table 10. Comparison of experimental results between HGASSO, SSO, and GA in terms of mean, standard deviation, maximam value, median, and best fitness for the Goldstein and Price (GP) test function. The bold font with the highlighted background represents the best fitness and mean for the respective benchmark function

\begin{tabular}{|c|c|c|c|c|c|c|c|}
\hline Algorithm & Iteration no. & Average, mean & Std. & Max. & Median & Best fitness \\
\hline \multirow{6}{*}{ HGASSO } & 30 & $\mathbf{7 . 9 6 6 9}$ & 13994.6643 & 59.90158 & 3.009872 & $\mathbf{3}$ \\
\cline { 2 - 7 } & 100 & $\mathbf{5 . 0 2 8 1}$ & 24078.6216 & 90.36963 & 3 & $\mathbf{3}$ \\
\cline { 2 - 8 } & 400 & $\mathbf{3 . 3 0 4 2}$ & 3045.3292 & 67.17167 & 3 & $\mathbf{3}$ \\
\cline { 2 - 8 } & 1000 & $\mathbf{4 . 2 4 3 2}$ & 610.4686 & 1154.375 & 3 & $\mathbf{3}$ \\
\hline \multirow{4}{*}{ SSO } & 30 & 346.1439 & 0.18953 & 75.95701 & 3 & $\mathbf{3}$ \\
\cline { 2 - 8 } & 100 & 710.5087 & 1.2212 & 15.27657 & 3.000677 & $\mathbf{3}$ \\
\cline { 2 - 8 } & 400 & 91.9061 & 1.4806 & 31.75424 & 3 & $\mathbf{3}$ \\
\cline { 2 - 8 } & 1000 & 13.9474 & 0.048025 & 4.277457 & 3 & $\mathbf{3}$ \\
\hline \multirow{4}{*}{ GA } & 30 & 18301161.1854 & 0.023637 & 64052.63 & 5.059146 & $\mathbf{3}$ \\
\cline { 2 - 8 } & 100 & 1264107.5031 & 93246.1569 & 689269.2 & 3 & $\mathbf{3}$ \\
\cline { 2 - 8 } & 400 & 69346.2974 & 0.18467 & 3.999575 & 3 & $\mathbf{3}$ \\
\hline
\end{tabular}

Figure 20. Comparison of experimental results between HGASSO, SSO, and GA in term of convergence for the Goldstein and Price (GP) test function
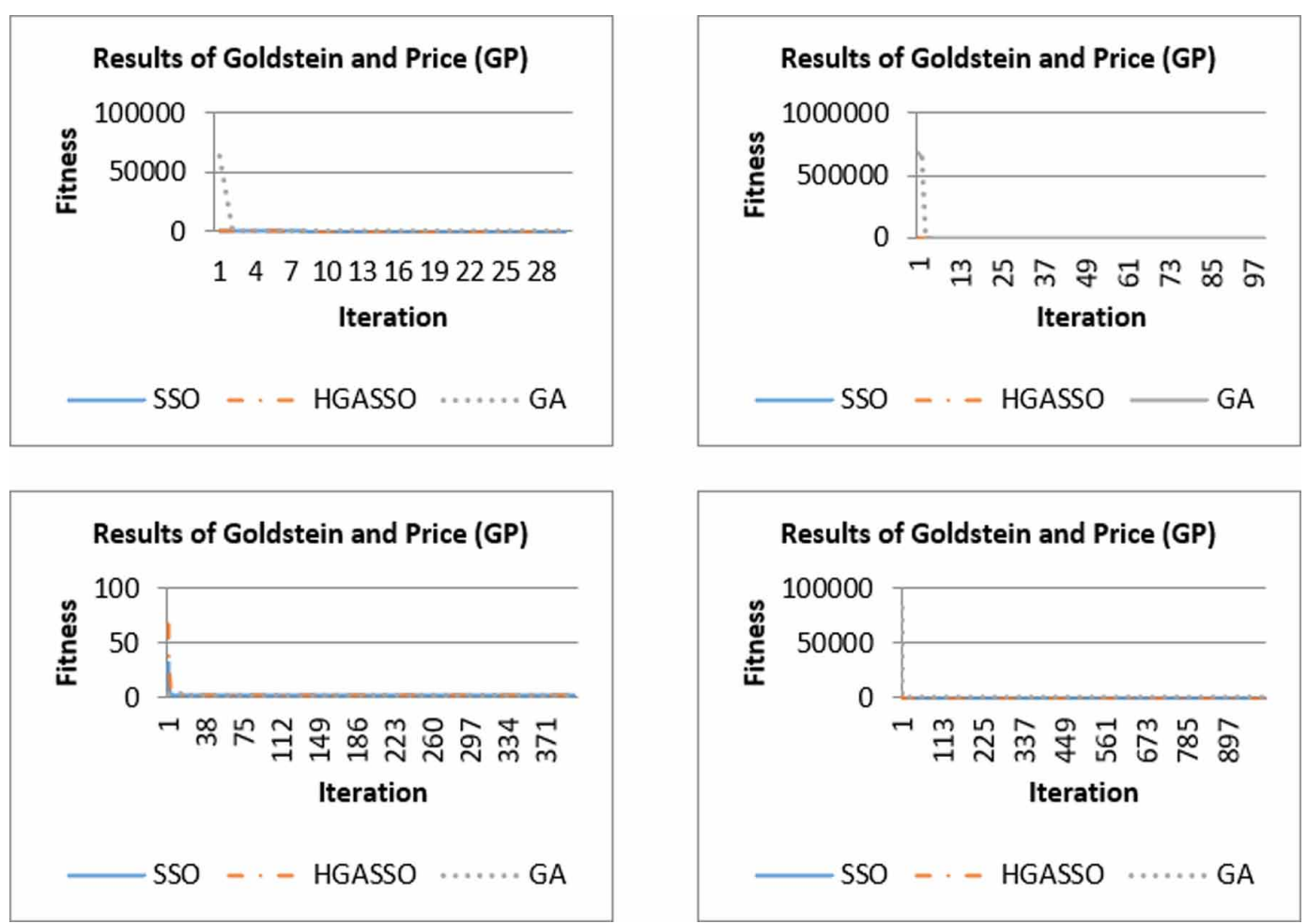
International Journal of Applied Metaheuristic Computing

Volume $13 \cdot$ Issue 1

Table 11. Comparison of experimental results between HGASSO, SSO, and GA in terms of mean, standard deviation, maximam value, median, and best fitness for the Shubert test function. The bold font with the highlighted background represents the best fitness and mean for the respective benchmark function

\begin{tabular}{|c|c|c|c|c|c|c|}
\hline Algorithm & Iteration no. & Average, mean & Std. & Max. & Median & Best fitness \\
\hline \multirow{6}{*}{ HGASSO } & 30 & $\mathbf{- 1 7 2 . 1 0 8 4}$ & 7.2895 & -181.491 & -186.711 & $\mathbf{- 1 8 6 . 7 3 0 9}$ \\
\cline { 2 - 7 } & 100 & $\mathbf{- 1 7 5 . 6 0 1 7}$ & 4.5234 & -167.469 & -186.926 & $\mathbf{- 1 8 6 . 7 3 0 9}$ \\
\cline { 2 - 7 } & 400 & $\mathbf{- 1 8 3 . 1 6 2 4}$ & 15.3322 & -39.1642 & -186.7 & $\mathbf{- 1 8 6 . 7 3 0 9}$ \\
\cline { 2 - 7 } & 1000 & $\mathbf{- 1 8 6 . 2 0 4 6}$ & 2.6593 & -114.26 & -186.707 & $\mathbf{- 1 8 6 . 7 3 0 9}$ \\
\hline \multirow{4}{*}{ SSO } & 30 & -83.8316 & 28.3472 & -38.3897 & -186.728 & $\mathbf{- 1 8 6 . 7 3 0 9}$ \\
\cline { 2 - 7 } & 100 & -167.5589 & 6.8631 & -126.795 & -186.731 & $\mathbf{- 1 8 6 . 7 3 0 9}$ \\
\cline { 2 - 7 } & 400 & -179.9879 & 15.2599 & -71.217 & -186.731 & $\mathbf{- 1 8 6 . 7 3 0 9}$ \\
\cline { 2 - 7 } & 1000 & -181.9694 & 6.2583 & -73.0234 & -186.731 & $\mathbf{- 1 8 6 . 7 3 0 9}$ \\
\hline \multirow{6}{*}{ GA } & 30 & -100.130 & 11.0655 & -132.217 & -186.411 & -186.1020 \\
\cline { 2 - 7 } & 100 & -164.6632 & 28.7466 & -75.3547 & -185.645 & -186.5432 \\
\cline { 2 - 7 } & 400 & -175.7541 & 22.8959 & -26.1109 & -186.731 & $\mathbf{- 1 8 6 . 7 3 0 9}$ \\
\cline { 2 - 7 } & 1000 & -185.0282 & 5.742 & -79.429 & -186.731 & $\mathbf{- 1 8 6 . 7 3 0 9}$ \\
\hline
\end{tabular}

Figure 21. Comparison of experimental results between HGASSO, SSO, and GA in term of convergence for the Shubert test function
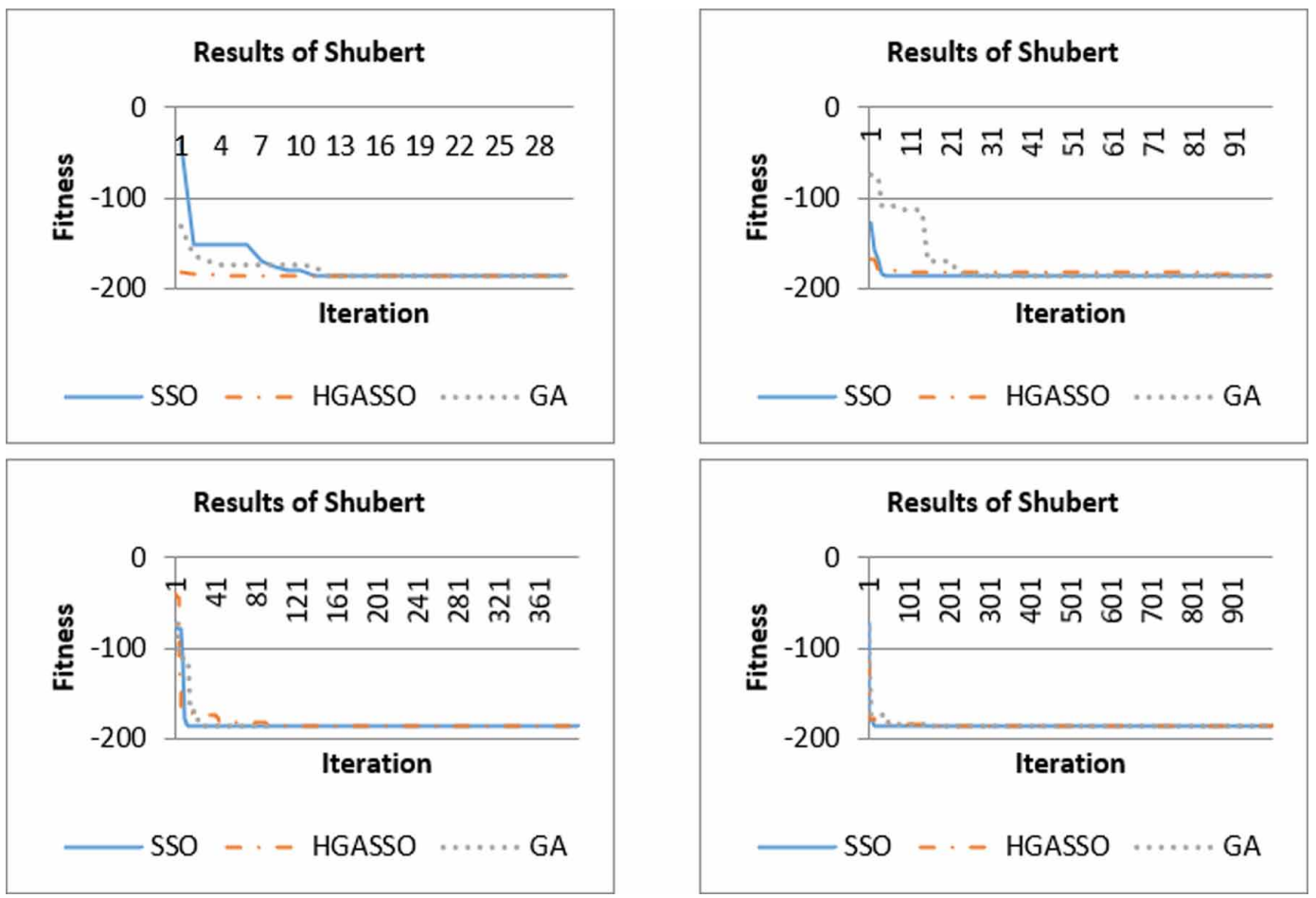
Table 12. Comparison of experimental results between HGASSO, SSO, and GA in terms of mean, standard deviation, maximam value, median, and best fitness for the Shekel test function. The bold font with the highlighted background represents the best fitness and mean for the respective benchmark function

\begin{tabular}{|c|c|c|c|c|c|c|}
\hline Algorithm & Iteration no. & Average, mean & Std. & Max. & Median & Best fitness \\
\hline \multirow{4}{*}{ HGASSO } & 30 & $\mathbf{- 6 . 8 2 9 6}$ & 2.1785 & -0.47689 & -7.37539 & $\mathbf{- 8 . 1 0 9}$ \\
\cline { 2 - 7 } & 100 & $\mathbf{- 7 . 7 2 0 6}$ & 1.336 & -0.70059 & -8.06945 & $\mathbf{- 8 . 1 0 1 3}$ \\
\cline { 2 - 7 } & 400 & $\mathbf{- 5 . 0 1 4 4}$ & 0.29884 & -0.42681 & -4.04711 & $\mathbf{- 8 . 6 4 7 1}$ \\
\cline { 2 - 7 } & 1000 & -10.1939 & 0.54918 & -0.4664 & -10.2329 & $\mathbf{- 1 0 . 5}$ \\
\hline \multirow{4}{*}{ SSO } & 30 & -3.0108 & 0.88317 & -1.20946 & -4.06778 & -4.0699 \\
\cline { 2 - 7 } & 100 & -3.8949 & 0.40467 & -0.97569 & -4.0687 & -4.066 \\
\cline { 2 - 7 } & 400 & -4.0436 & 0.097992 & -2.14548 & -4.0687 & -4.0687 \\
\cline { 2 - 7 } & 1000 & $\mathbf{- 1 0 . 4 3 6 8}$ & 0.3629 & -3.40547 & -10.5 & $\mathbf{- 1 0 . 5}$ \\
\hline \multirow{4}{*}{ GA } & 30 & -1.8848 & 0.8852 & -0.48731 & -2.19278 & -3.5802 \\
\cline { 2 - 7 } & 100 & -3.6846 & 0.85376 & -0.19777 & -4.0617 & -4.0617 \\
\cline { 2 - 7 } & 400 & -3.9919 & 0.32832 & -0.37066 & -4.05631 & -4.0775 \\
\hline
\end{tabular}

Figure 22. Comparison of experimental results between HGASSO, SSO, and GA in term of convergence for the Shekel test function
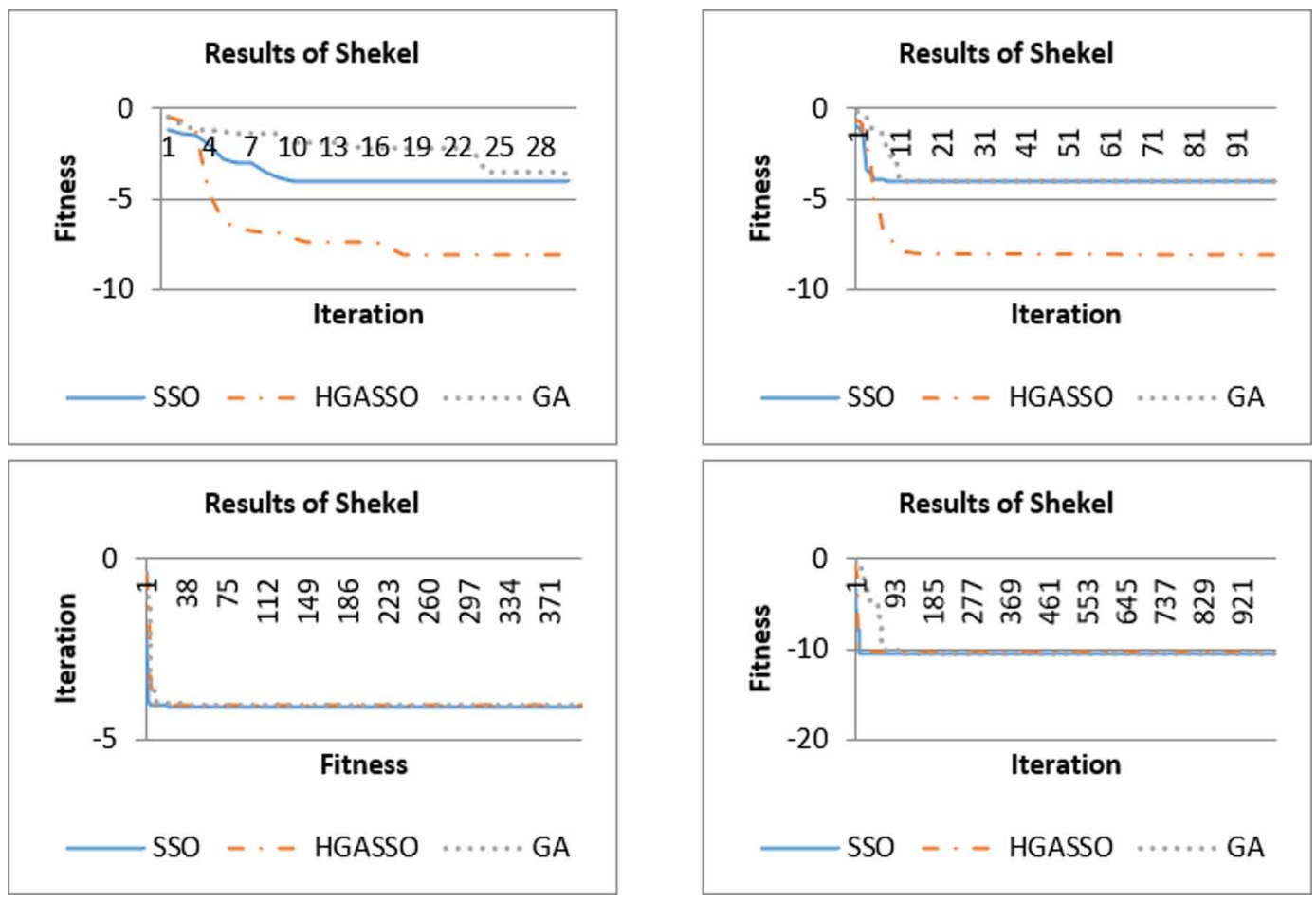
Table 13. Ranking the algorithms from best to worse obtained fitness value

\begin{tabular}{|c|c|}
\hline Function & $\begin{array}{c}\text { The approaches are ranked from the best-obtained } \\
\text { fitness value to the worse obtained fitness value }\end{array}$ \\
\hline Rastrigin & HGASSO, SSO, GA \\
\hline Ackley & HGASSO, SSO, GA \\
\hline Bukin & HGASSO, SSO, GA \\
\hline Griewank & HGASSO, GA, SSO \\
\hline Cross-in-Tray & All in the same rank \\
\hline Levy function N.13 & HGASSO, SSO, GA \\
\hline Branin & All in the same rank \\
\hline Easom & HGASSO and SSO in the first rank, followed by GA \\
\hline Goldstein and Price (GP) & All in the same rank \\
\hline Shubert & HGASSO and SSO in the first rank, followed by GA \\
\hline Shekel & HGASSO, SSO, GA \\
\hline
\end{tabular}

Table 14, HGASSO significantly outperforms GA in solving the majority of benchmarks functions. This is clear in the $p$-Value and mean difference between HGASSO and the other approaches, which is significant at the 0.05 level.

At the end, comparisons between SSO, GA, and HGASSO can be summarized as in Table 15 (Shehadeh et al., 2017; Shehadeh et al., 2018c).

\section{DISCUSSION}

Multimodal problems include a set of mathematical operations in their evaluation, such as cosine, sine, exponent, and multiplications of multiple variables. Furthermore, these problems mainly have several local extremes, which many optimization algorithms are trapped in one of their several local minima. Based on that, many local search approaches have been hybridized with evolutionary algorithms to skip these local minima easily until obtaining the optimal solution of global minima. The concept of the hybrid algorithm is usually used to combine the concept of the evolutionary method with the concept of local search in one procedure. SSO method is a promising optimization approach that is inspired by sperm motility. This method is suitable for solving variant types of continuous nonlinear functions, but until recently it had not been hybridized with evolutionary algorithms to deal with multimodal problems.

In this paper, HGASSO, SSO, and GA have been evaluated on multimodal test bed suites. Standard metrics are calculated in the experiments, namely the best fitness value, mean value, median, maximum value, standard deviation and the algorithm convergence. In addition, the statistical test, called "one-way ANOVA (Tukey's test)" is adopted in this study to compare between approaches. For each approach evaluated in the experiment, the maximum iteration for each benchmark function is set to $30,100,400$, and 1000 respectively. The evaluation is reaccredited 10 times for each benchmark test function to ensure the convergence of the results.

Overall, the proposed HGASSO algorithm outperformed both SSO and GA algorithms in solving benchmarks functions that include the cosine, sine, and exponent in their evaluation. HGASSO outperformed SSO and GA in solving the Rastrigin, Ackley, Bukin, Griewank, Levy function N.13, and Shekel multimodal functions, and obtained better fitness value than GA of Easom and Shubert multimodal functions. Additionally, the high-quality performance of the proposed HGASSO was 
Table 14. Analysis of "one-way ANOVA (Tukey's test)" between SSO, GA and HGASSO for all benchmarks functions

\begin{tabular}{|c|c|c|c|c|}
\hline Benchmark function & $\begin{array}{l}\text { Algorithm } \\
\text { (I) }\end{array}$ & $\begin{array}{c}\text { Algorithm } \\
\text { (J) }\end{array}$ & $\begin{array}{c}\text { Mean } \\
\text { Difference } \\
\text { (I-J) }\end{array}$ & p-Value \\
\hline \multirow[t]{2}{*}{ Rastrigin } & HGASSO & GA & -0.244169 & 0.043378 \\
\hline & & SSO & -0.006159 & $<0.001 *$ \\
\hline \multirow[t]{2}{*}{ Ackley } & HGASSO & GA & -1.7273322 & $<0.001 *$ \\
\hline & & SSO & -0.0023981 & $<0.001 *$ \\
\hline \multirow[t]{2}{*}{ Bukin } & HGASSO & GA & -0.43538 & $<0.001 *$ \\
\hline & & SSO & -0.48207 & 0.794144 \\
\hline \multirow[t]{2}{*}{ Griewank } & HGASSO & GA & -0.0095911 & $<0.001^{*}$ \\
\hline & & SSO & -0.3452741 & 0 \\
\hline \multirow[t]{2}{*}{ Cross-in-Tray } & HGASSO & GA & -0.0005 & 0.240279 \\
\hline & & SSO & 0 & 0.654482 \\
\hline \multirow[t]{2}{*}{ Levy function N.13 } & HGASSO & GA & -0.09938755 & 0.096145 \\
\hline & & SSO & -1.02799055 & 0.720205 \\
\hline \multirow[t]{2}{*}{ Branin } & HGASSO & GA & -0.17267 & 0.127417 \\
\hline & & SSO & -0.03076 & 0.197382 \\
\hline \multirow[t]{2}{*}{ Easom } & HGASSO & GA & -0.06765 & 0 \\
\hline & & SSO & -0.01466 & 0.012605 \\
\hline \multirow[t]{2}{*}{ Goldstein and Price (GP) } & HGASSO & GA & -77241.1859 & 0.288343 \\
\hline & & SSO & -9.7042 & 0.122608 \\
\hline \multirow[t]{2}{*}{ Shubert } & HGASSO & GA & -1.1764 & $<0.001 *$ \\
\hline & & SSO & -4.2352 & 0.363168 \\
\hline \multirow[t]{2}{*}{ Shekel } & HGASSO & GA & 0.0521 & 0.017848 \\
\hline & & SSO & 0.2429 & $<0.001 *$ \\
\hline
\end{tabular}

reflected on the metrics of convergence in which attained a typical convergence regarding to these benchmark functions. This proves that the proposed HGASSO has a better convergence than the other algorithms to explore the domain of search space and to skip the multiple local minima easily.

\section{CONCLUSION}

In this article, a hybrid approach consists of two methods has been proposed to solve multimodal benchmark functions. This method integrates the concept of discreet procedures of the evolutionary method that is originally inspired by GA with the concept of local search of SSO. The proposed approach is validated in two ways. First, we used the standard multimodal problems that are currently adopted in the evolutionary single objective optimization community. The fitness value, average, maximum value, median value and standard deviation have been chosen to compare between the proposed algorithm and other algorithms based on a set of generation sizes, such as 30, 100, 400, and 1000. Furthermore, a statistical test, called "one-way ANOVA (Tukey's test)" is used in this study to compare between approaches. Second, the algorithm convergence of each algorithm for 
Table 15. Comparison between SSO, GA, and HGASSO (Shehadeh et al., 2017; Shehadeh et al., 2018c)

\begin{tabular}{|c|c|c|c|}
\hline $\begin{array}{l}\text { Criteria of } \\
\text { comparison }\end{array}$ & SSO & GA & HGASSO \\
\hline Type of procedure & Continuous procedure. & Discrete procedure. & Hybrid procedure. \\
\hline Type of a metaphor & $\begin{array}{l}\text { Mimics the motility } \\
\text { of swarm of sperm } \\
\text { through the natural } \\
\text { fertilization procedure } \\
\text { of an egg. }\end{array}$ & $\begin{array}{l}\text { Mimics the theory of evolution } \\
\text { applied to biology that proposed by } \\
\text { Darwin. This procedure simulates } \\
\text { the structure of the chromosome and } \\
\text { its evolution. }\end{array}$ & $\begin{array}{l}\text { Mimics the theory of } \\
\text { evolution applied to biology } \\
\text { that proposed by Darwin } \\
\text { as well as it uses the local } \\
\text { search procedure of SSO. }\end{array}$ \\
\hline $\begin{array}{l}\text { Sorting the } \\
\text { population }\end{array}$ & $\begin{array}{l}\text { Population will not be } \\
\text { sorted. }\end{array}$ & $\begin{array}{l}\text { Population needs ranking and } \\
\text { selection. }\end{array}$ & $\begin{array}{l}\text { The population will be sorted } \\
\text { at the end of the procedure } \\
\text { to determine the global best } \\
\text { solution. }\end{array}$ \\
\hline $\begin{array}{l}\text { Use crossover } \\
\text { operation }\end{array}$ & $\begin{array}{c}\text { No need for crossover } \\
\text { Operation. }\end{array}$ & Apply crossover operation. & Apply crossover operation. \\
\hline $\begin{array}{l}\text { Use mutation } \\
\text { operation }\end{array}$ & No need for mutation. & Apply mutation operation. & Apply mutation operation. \\
\hline $\begin{array}{l}\text { Best solution effects } \\
\text { Population }\end{array}$ & $\begin{array}{l}\text { The value of winner } \\
\text { (the best solution) is } \\
\text { used as a reference } \\
\text { value for the swarm to } \\
\text { modify their velocities. }\end{array}$ & $\begin{array}{c}\text { Treat with every individual } \\
\text { independently. }\end{array}$ & $\begin{array}{l}\text { Apply a local search strategy } \\
\text { of SSO to enhance the } \\
\text { results. }\end{array}$ \\
\hline
\end{tabular}

each benchmark function has been drawn, after that, compared based on different generation sizes. The results of these comparisons demonstrated that HGASSO synthesizes the merits of both SSO and GA, and it is yet effective model and simple to handle various types of continuous optimization problems. Moreover, the experimental results show that the proposed HGASSO is a viable alternative since HGASSO has a good ability to solve the multimodal benchmark functions, which is superior to the other approaches in the ability to reaching the global optimum easily.

The benchmarks models in this study may have their limitations, which other metrics in real experiments may affect the outcomes of the studies. Hence, in the future, the proposed HGASSO should be implemented to solve real problems that utilize cosine, sine, and exponent in there mathematical modeling such as minimizing propagation loss and interference of wireless, telecommunication and mobile networks (Shehadeh et al., 2020; Hamdan et al., 2015; Hamdan, 2018), engineering applications (Jayabarathi et al., 2018), designing the optimal spacing and length of Yagi-Uda antenna (Baumgartner et al., 2017). Furthermore, in the future, the proposed HGASSO should be compared against a set of well-known approaches, such as "Chaotic Gravitational Search Algorithm (CGSA)" (Rather \& Bala, 2020), "Grey Wolf Optimizer (GWO)" (Mirjalili et al., 2014), and "Sine Cosine Algorithm (SCA)" (Mirjalili et al., 2016). 


\section{REFERENCES}

Adorio, E. P., \& Diliman, U. (2005). Mvf-multivariate test functions library in c for unconstrained global optimization. Quezon City. Metro Manila, Philippines, 4, 100-104.

Back, T. (1996). Evolutionary algorithms in theory and practice: evolution strategies, evolutionary programming, genetic algorithms. Oxford University Press. doi:10.1093/oso/9780195099713.001.0001

Banharnsakun, A., Achalakul, T., \& Sirinaovakul, B. (2011). The best-so-far selection in artificial bee colony algorithm. Applied Soft Computing, 11(2), 2888-2901. doi:10.1016/j.asoc.2010.11.025

Baumgartner, P., Bauernfeind, T., Biro, O., Hackl, A., Magele, C., Renhart, W., \& Torchio, R. (2017). Multiobjective optimization of Yagi-Uda antenna applying enhanced firefly algorithm with adaptive cost function. IEEE Transactions on Magnetics, 54(3), 1-4. doi:10.1109/TMAG.2017.2764319

Chaudhary, D., \& Kumar, B. (2019). Cost optimized hybrid genetic-gravitational search algorithm for load scheduling in cloud computing. Applied Soft Computing, 83, 105627. doi:10.1016/j.asoc.2019.105627

Fang, N., Zhou, J., Zhang, R., Liu, Y., \& Zhang, Y. (2014). A hybrid of real coded genetic algorithm and artificial fish swarm algorithm for short-term optimal hydrothermal scheduling. International Journal of Electrical Power \& Energy Systems, 62, 617-629. doi:10.1016/j.jiepes.2014.05.017

Fogel, D. B. (2005). Evolutionary Computation: Toward a New Philosophy of Machine Intelligence (3rd ed.). Wiley-IEEE Press. doi:10.1002/0471749214

Gacem, A., \& Benattous, D. (2017). Hybrid genetic algorithm and particle swarm for optimal power flow with non-smooth fuel cost functions. International Journal of System Assurance Engineering and Management, 8(1), 146-153. doi:10.1007/s13198-014-0312-8

Gholami, A., Bonakdari, H., Ebtehaj, I., Mohammadian, M., Gharabaghi, B., \& Khodashenas, S. R. (2018). Uncertainty analysis of intelligent model of hybrid genetic algorithm and particle swarm optimization with ANFIS to predict threshold bank profile shape based on digital laser approach sensing. Measurement, 121, 294-303. doi:10.1016/j.measurement.2018.02.070

Goldberg, D. E., \& Holland, J. H. (1988). Genetic algorithms and machine learning. Machine Learning, 3(2/3), 95-99. doi:10.1023/A:1022602019183

Hamdan, M., Bani-Yaseen, M., \& Shehadeh, H. A. (2018). Multi-objective optimization modeling for the impacts of 2.4-GHz ISM band interference on IEEE 802.15. 4 health sensors. In L. Ismail \& L. Zhang (Eds.), Information Innovation Technology in Smart Cities (pp. 317-330). Springer. doi:10.1007/978-981-10-1741-4_21

Hamdan, M., Yassein, M. B., \& Shehadeh, H. A. (2015). Multi-objective optimization modeling of interference in home health care sensors. 11th international conference on innovations in information technology (IIT), 219-224. doi:10.1109/INNOVATIONS.2015.7381543

Jayabarathi, T., Raghunathan, T., \& Gandomi, A. H. (2018). The bat algorithm, variants and some practical engineering applications: a review. In Nature-Inspired Algorithms and Applied Optimization. Springer. doi:10.1007/978-3-319-67669-2_14

Kaipa, K. N., \& Ghose, D. (2017). Multimodal function optimization. In K. N. Kaipa \& D. Ghose (Eds.), Book: Glowworm Swarm Optimization (pp. 91-131). Springer. doi:10.1007/978-3-319-51595-3_4

Kao, Y. T., \& Zahara, E. (2008). A hybrid genetic algorithm and particle swarm optimization for multimodal functions. Applied Soft Computing, 8(2), 849-857. doi:10.1016/j.asoc.2007.07.002

Krzeszowski, T., \& Wiktorowicz, K. (2016). Evaluation of selected fuzzy particle swarm optimization algorithms. In Federated Conference on Computer Science and Information Systems (FedCSIS). IEEE. doi:10.15439/2016F206

Langdon, W. B., \& Poli, R. (2002). Foundations of Genetic Programming (3rd ed.). Springer Science \& Business Media. doi:10.1007/978-3-662-04726-2

Mirjalili, S. (2016). SCA: A sine cosine algorithm for solving optimization problems. Knowledge-Based Systems, 96, 120-133. doi:10.1016/j.knosys.2015.12.022 
Mirjalili, S., Mirjalili, S. M., \& Lewis, A. (2014). Grey wolf optimizer. Advances in Engineering Software, 69, 46-61. doi:10.1016/j.advengsoft.2013.12.007

Moin, N. H., Chung Sin, O., \& Omar, M. (2015). Hybrid genetic algorithm with multiparents crossover for job shop scheduling problems. Mathematical Problems in Engineering, 12, 1-12. doi:10.1155/2015/210680

Molga, M., \& Smutnicki, C. (2005). Test functions for optimization needs. Majid Vafaei Jahan Web. https://www. vafaeijahan.com/en/wp-content/uploads/2012/02/Test-functions-for-optimization-needs.pdf

Paulinas, M., \& Ušinskas, A. (2007). A survey of genetic algorithms applications for image enhancement and segmentation. Inf. Technol. Information Technology and Control, 36, 278-284. https://itc.ktu.lt/index.php/ITC/ article/view/11886

Pomar, L. A., Pulido, E. C., \& Roa, J. D. T. (2017). A hybrid genetic algorithm and particle swarm optimization for flow shop scheduling problems. In 4th Workshop on Engineering Applications, WEA. Springer.

Rather, S. A., \& Bala, P. S. (2020). Swarm-based chaotic gravitational search algorithm for solving mechanical engineering design problems. World Journal of Engineering, 17(1), 97-114. doi:10.1108/WJE-09-2019-0254

Rbouh, I., \& El Imrani, A. A. (2014). Hurricane Search algorithm a new model for function optimization. In 2014 5th International Conference on Information and Communication Systems (ICICS). IEEE. doi:10.1109/ IACS.2014.6841947

Samuel, G. G., \& Rajan, C. C. A. (2015). Hybrid: Particle swarm optimization-genetic algorithm and particle swarm optimization-shuffled frog leaping algorithm for long-term generator maintenance scheduling. International Journal of Electrical Power \& Energy Systems, 65, 432-442. doi:10.1016/j.ijepes.2014.10.042

Saravanan, R., \& Sachithanandam, M. (2001). Genetic algorithm (GA) for multivariable surface grinding process optimisation using a multi-objective function model. International Journal of Advanced Manufacturing Technology, 17(5), 330-338. doi:10.1007/s001700170167

Sathya, S. S., \& Radhika, M. V. (2013). Convergence of nomadic genetic algorithm on benchmark mathematical functions. Applied Soft Computing, 13(5), 2759-2766. doi:10.1016/j.asoc.2012.11.011

Shehadeh, H. A., Ahmedy, I., \& Idris, M. Y. I. (2018). Sperm swarm optimization algorithm for optimizing wireless sensor network challenges. Proceedings of the ACM International Conference on Communications and Broadband Networking (ICCBN), 53-59. doi:10.1145/3193092.3193100

Shehadeh, H. A., Ahmedy, I., \& Idris, M. Y. I. (2018). Empirical study of sperm swarm optimization algorithm. In Proceedings of SAI Intelligent Systems Conference. Springer. doi:10.1007/978-3-030-01057-7_80

Shehadeh, H. A., Idna Idris, M. Y., Ahmedy, I., Ramli, R., \& Mohamed Noor, N. (2018). The Multi-objective optimization algorithm based on sperm fertilization procedure (MOSFP) method for solving wireless sensor networks optimization problems in smart grid applications. Energies, 11(1), 97. doi:10.3390/en11010097

Shehadeh, H. A., Idris, M. Y. I., Ahmedy, I., \& Hassen, H. R. (2020). Optimal placement of near ground VHF/ UHF radio communication network as a multi objective problem. Wireless Personal Communications, 110(3), 1169-1197. doi:10.1007/s11277-019-06780-6

Shehadeh, H. A., ldris, M., \& Ahmedy, I. (2017). Multi-objective optimization algorithm based on sperm fertilization procedure (MOSFP). Symmetry, 9(10), 241. doi:10.3390/sym9100241

Shi, Y., \& Eberhart, R. C. (1999). Empirical study of particle swarm optimization. IEEE Proceedings of the 1999 Congress on Evolutionary Computation-CEC99, 1945-1950. doi: doi:10.1109/CEC.1999.785511

Soleimani, H., \& Kannan, G. A. (2015). A hybrid particle swarm optimization and genetic algorithm for closedloop supply chain network design in large-scale networks. Applied Mathematical Modelling, 39(14), 3990-4012. doi:10.1016/j.apm.2014.12.016

Surjanovic, S., \& Bingham, D. (2013). Virtual library of simulation experiments: test functions and datasets. https://www.sfu.ca/ ssurjano/optimization.html 
Hisham A. Shehadeh received the B.S. degree in computer science from Al-Balqa 'Applied University, Jordan, in 2012, the M.S. degree in computer science from the Jordan University of Science and Technology, Irbid, Jordan, in 2014, and the Ph.D. degree from the Department of Computer System and Technology, University of Malaya (UM), Kuala Lumpur, Malaysia, in 2018. He was a Research Assistant at UM from 2017 to 2018. He was a Teaching Assistant and a Lecturer with CS Department, College of Computer and Information Technology, Jordan University of Science and Technology from 2013 to 2014 and from 2014 to 2016 respectively. Currently, He is an Assistant Professor of Computer Science at the departments of Computer Science and Computer Information System, Amman Arab University, Amman, Jordan. His current research interests are, Intelligent Computing, Metaheuristic Algorithms and Algorithmic Engineering Applications of Wireless Networks.

Hossam M. J. Mustafa received his PhD degree in Computer Science from Universiti Kebangsaan, Malaysia in 2019. He is an Assistant Professor of Computer Science at the departments of Computer Science and Computer Information System, Amman Arab University, Amman, Jordan. His current research interests are Intelligent Computing, Metaheuristic Algorithms and Machine Learning.

Mohammad Tubishat received his PhD degree in Computer Science (Artificial Intelligence - Natural Language Processing) from the University of Malaya in 2019. In addition, MSc in Computer and Information Sciences from Yarmouk University in 2004. Furthermore, BSc degree in Computer Science from Yarmouk University in 2002. His research interests include natural language processing, data mining, artificial intelligence, machine learning, optimization algorithms, data science, cybersecurity, and sentiment analysis. He is now working as a lecturer (Assistant Professor) at Asia Pacific University of Technology and innovation. 